\title{
A new radio molecular line survey of planetary nebulae $\mathrm{HNC} / \mathrm{HCN}$ as a diagnostic of ultraviolet irradiation
}

\author{
J. Bublitz ${ }^{1,2}$, J. H. Kastner ${ }^{2}$, M. Santander-García ${ }^{3}$, V. Bujarrabal ${ }^{3}$, J. Alcolea ${ }^{3}$, and R. Montez Jr. ${ }^{4}$ \\ ${ }^{1}$ Institut de Planétologie et d'Astrophysique de Grenoble (IPAG) UMR 5274, 38041 Grenoble, France \\ ${ }^{2}$ School of Physics \& Astronomy, Center for Imaging Science, Laboratory for Multiwavelength Astrophysics, Rochester Institute of \\ Technology, 54 Lomb Memorial Drive, Rochester, NY 14623, USA \\ e-mail: jtb1435@rit.edu \\ 3 Observatorio Astronómico Nacional, Alfonso XII, 3, 28014 Madrid, Spain \\ ${ }^{4}$ Smithsonian Astrophysical Observatory, 60 Garden Street, Cambridge, MA 02138, USA
}

Received 8 October 2018 / Accepted 7 April 2019

\begin{abstract}
Certain planetary nebulae (PNe) contain shells, filaments, or globules of cold gas and dust whose heating and chemistry are likely driven by UV and X-ray emission from their central stars and from wind-collision-generated shocks. We present the results of a survey of molecular line emission in the $88-236 \mathrm{GHz}$ range from nine nearby $(<1.5 \mathrm{kpc})$ planetary nebulae spanning a range of UV and X-ray luminosities, using the $30 \mathrm{~m}$ telescope of the Institut de Radioastronomie Millimétrique. Rotational transitions of thirteen molecules, including $\mathrm{CO}$ isotopologues and chemically important trace species, were observed and the results compared with and augmented by previous studies of molecular gas in $\mathrm{PNe}$. Lines of the molecules $\mathrm{HCO}^{+}, \mathrm{HNC}, \mathrm{HCN}$, and $\mathrm{CN}$, which were detected in most objects, represent new detections for four planetary nebulae in our study. Specifically, we present the first detections of ${ }^{13} \mathrm{CO}(1-0,2-1), \mathrm{HCO}^{+}$, $\mathrm{CN}, \mathrm{HCN}$, and $\mathrm{HNC}$ in $\mathrm{NGC} 6445 ; \mathrm{HCO}^{+}$in $\mathrm{BD}+30^{\circ} 3639 ;{ }^{13} \mathrm{CO}(2-1), \mathrm{CN}, \mathrm{HCN}$, and $\mathrm{HNC}$ in NGC 6853 ; and ${ }^{13} \mathrm{CO}(2-1)$ and $\mathrm{CN}$ in NGC 6772. Flux ratios were analyzed to identify correlations between the central star and/or nebular UV and X-ray luminosities and the molecular chemistries of the nebulae. This analysis reveals a surprisingly robust dependence of the HNC/HCN line ratio on PN central star UV luminosity. There exists no such clear correlation between PN X-rays and various diagnostics of PN molecular chemistry. The correlation between $\mathrm{HNC} / \mathrm{HCN}$ ratio and central star UV luminosity demonstrates the potential of molecular emission line studies of PNe for improving our understanding of the role that high-energy radiation plays in the heating and chemistry of photodissociation regions.
\end{abstract}

Key words. astrochemistry - ISM: molecules - planetary nebulae: general - radio lines: ISM

\section{Introduction}

Planetary nebulae (PNe) arise from outflowing stellar mass during the late evolutionary stages of intermediate-mass stars $\left(\sim 0.8-8.0 M_{\odot}\right)$. These stars represent a significant stellar population in the Galaxy (Blöcker 2001; Edwards et al. 2014). They progress through the main sequence and red giant branch (RGB) evolutionary phases, where core $\mathrm{H}$ and $\mathrm{He}$ fusion occur respectively, into the shell-burning asymptotic giant branch (AGB) phase. Once the star arrives there, slow AGB winds, originating in shocks and pulsations and driven by radiation pressure on dust, remove the bulk of the stellar envelope at mass loss rates of $10^{-7}-10^{-4} M_{\odot} \mathrm{yr}^{-1}$ (Zack \& Ziurys 2013; Blöcker 2001). The presence of a close companion star can accelerate and otherwise profoundly affect this mass-loss process and the evolution of the resulting envelope (e.g., De Marco \& Izzard 2017, and references therein).

When the AGB envelope depletes to a mass of $\sim 10^{-2} M_{\odot}$, mass loss ceases and the star progresses to the post-AGB, and soon after, it becomes the central star of a PN (CSPN). Here, the cold, dusty AGB envelope is suddenly exposed to the hot (30-200 kK) post-fusion core's ionizing UV and (in some cases) $\mathrm{X}$-ray emission, which photodissociates and ionizes the envelope gas. This newly ionized gas constitutes the PN. Fast winds from the pre-white dwarf star (or its companion) may also slam into the slower moving ejected envelope, generating shocks that heat plasma to temperatures exceeding $\sim 10^{6} \mathrm{~K}$ (Kastner et al. 2012, and references therein). Hot bubbles that form from this wind interaction have been found to be X-ray-luminous, thereby producing another ionization mechanism within the PN. Interactions from the winds that form the bubbles are also thought to continue shaping the nebula throughout its lifetime (Balick \& Frank 2002; Huarte-Espinosa et al. 2012). Atomic gas and dust within the nebula limit the penetration depth of incident UV photons from the CSPN, leaving the outer layers of the nebula insulated from them. Only higher energy (X-ray) photons, $>0.5 \mathrm{keV}$, can penetrate the dense, neutral gas of the PN to ionize the cold, molecule-rich outer shells (Tielens \& Hollenbach 1985).

Millimeter $\mathrm{CO}$ and infrared $\mathrm{H}_{2}$ lines gave the first view of PN molecular gas 3 decades ago and have remained commonplace probes into the shells of ejected mass since (Zuckerman \& Gatley 1988; Huggins \& Healy 1989; Bachiller et al. 1991; Huggins et al. 1996, and references therein). Due to its high abundance, low critical density, and the low excitation temperatures of its rotational transitions, $\mathrm{CO}$ is the most commonly observed and more widely utilized molecular species found in PNe (Huggins et al. 1996). Infrared lines of $\mathrm{H}_{2}$ are also observed from $\mathrm{PNe}$, with detections of $\mathrm{H}_{2}$ mainly confined to bipolar (axisymmetric) nebulae (Kastner et al. 1996; Zuckerman \& Gatley 1988, and references therein). The complex interplay of PN central star radiation and composition of the proto-PN 
Table 1. Summary of physical data for observed PNe and their central stars.

\begin{tabular}{|c|c|c|c|c|c|c|c|c|c|c|}
\hline Name & $\begin{array}{l}\text { Morphology } \\
\text { (F08/SMV11) }\end{array}$ & $\begin{array}{c}\text { Angular } \\
\text { radius }^{(2)}\left({ }^{\prime \prime}\right)\end{array}$ & $\begin{array}{c}D \\
(\mathrm{kpc})\end{array}$ & $\begin{array}{c}R \\
(\mathrm{pc})\end{array}$ & $\begin{array}{c}\text { Age } \\
\left(10^{3} \mathrm{yr}\right)\end{array}$ & $\begin{array}{l}\text { Ionized PN } \\
\text { Mass }\left(M_{\odot}\right)\end{array}$ & $\begin{array}{l}\text { CSPN } \\
\text { sp type }\end{array}$ & $\begin{array}{c}T_{\star} \\
(\mathrm{kK})\end{array}$ & $\begin{array}{c}\text { Mass }_{\mathrm{CS}} \\
\left(M_{\odot}\right)\end{array}$ & Refs. \\
\hline BD+303639 & Er/Ecsarh & 4 & 1.52 & 0.02 & 0.8 & 0.01 & [WC9] & 32 & 0.58 & $a, b, c$ \\
\hline NGC 7027 & Bs/Mctspih & 14 & 0.92 & 0.03 & 0.7 & 0.05 & $\ldots$ & 175 & 0.67 & $\mathrm{c}, \mathrm{d}$ \\
\hline NGC 6445 & $\mathrm{Bs} / \mathrm{Mpi}$ & 17 & 1.38 & 0.14 & 5.3 & 0.18 & $\ldots$ & 170 & 0.64 & $e, f, c$ \\
\hline NGC 7008 & Efp/Bs & 43 & 0.97 & 0.15 & 5.7 & 0.08 & $\mathrm{O}(\mathrm{H})$ & 97 & 0.55 & $\mathrm{a}, \mathrm{g}, \mathrm{c}$ \\
\hline NGC 6720 (M 57) & Ebmr(h)/Ecsh & 35 & 0.70 & 0.13 & 7.0 & 0.09 & $\operatorname{hgO}(\mathrm{H})$ & 112 & 0.66 & $\mathrm{a}, \mathrm{h}$ \\
\hline NGC 6853 (M 27) & $\operatorname{Ebm}(\mathrm{h}) / \mathrm{Bbpih}$ & 49 & 0.38 & 0.37 & 10.0 & 0.41 & DAO & 114 & 0.63 & $\mathrm{a}, \mathrm{i}, \mathrm{j}$ \\
\hline NGC 6772 & $\mathrm{Ep} / \mathrm{E}$ & 32 & 1.27 & 0.22 & 10.9 & 0.17 & $\ldots$ & 135 & 0.64 & $\mathrm{e}, \mathrm{k}, \mathrm{c}$ \\
\hline NGC 7293 & $\operatorname{Bams}(\mathrm{h}) / \mathrm{Ltspir}$ & 402 & 0.20 & 0.46 & 16.3 & 0.35 & DAO & 107 & 0.63 & $\mathrm{i}, \mathrm{k}, \mathrm{a}$ \\
\hline NGC 6781 & Bam(h:)/Bth & 53 & 0.95 & 0.32 & 20.0 & 0.44 & DAO & 112 & 0.57 & $\mathrm{a}, \mathrm{l}, \mathrm{c}$ \\
\hline
\end{tabular}

Notes. ${ }^{(1)}$ Morphology descriptions as defined in Frew (2008) (F08); a: asymmetry present, B: bipolar, b: bipolar core, E: elliptical, f: filled amorphous center, (h): distinct outer halo, m: multiple shells, p: point symmetry present, r: dominant ring structure, s: internal structure. Morphology description modified slightly from Sahai et al. (2011) (SMV11); a: ansae, B: bipolar, c: closed outer lobes, E: elongated, h: halo, i: inner bubble, L: collimated lobe pair, M: multipolar, p: point symmetry, r: radial rays, s: CSPN apparent, t: bright central toroidal structure. ${ }^{(2)}$ Angular radii estimated using DSS data.

References. For PN data: a: Frew et al. (2016), b: Li et al. (2002), c: Kastner et al. (2012), d: Latter et al. (2000), e: Stanghellini \& Shaw (2008), f: Phillips (1984), g: Górny et al. (1997), h: O’Dell et al. (2007), i: Gaia Collaboration (2018), j: O’Dell et al. (2002), k: Ali et al. (2012), 1: Ueta et al. (2014).

produce a rich environment for molecular chemistry that has served as motivation for various radio molecular line surveys (e.g., Bachiller et al. 1997; Zhang et al. 2008; Edwards et al. 2014; Schmidt et al. 2018). Whereas the foregoing surveys were largely restricted to well-studied objects, the recent molecular line surveys of Schmidt \& Ziurys (2016, 2017a,b) extended the PN sample coverage to younger objects.

In this paper we present molecular line surveys of nine PNe, obtained with the Institut de Radioastronomie Millimétrique (IRAM) $30 \mathrm{~m}$ telescope within the frequency range 88-236 $\mathrm{GHz}$. These observations were specifically intended to explore the utility of potential tracers of high-energy irradiation of molecular gas. We report new detections of molecules and/or molecular transitions in four of these $\mathrm{PNe}$, and place these results in the context of previously reported PN molecular line detections and measurements. We then evaluate the integrated flux ratios of observed molecular lines to study correlations between the molecular chemistry and high-energy radiation properties of the CSPNe. Specifically, we strive to find tracers of non-LTE chemistry due to X-irradiation of molecular gas and the effects of CSPN UV emission on the photodissociation regions (PDR) within PNe.

\section{Observations and data reduction}

The sample of nine PNe studied here were selected from the larger Chandra Planetary Nebula Survey PN sample (Kastner et al. 2012; Freeman et al. 2014). The ChanPlaNs survey targeted 35 objects within $1.5 \mathrm{kpc}$ of Earth with the aim to identify and categorize PN X-ray emission, so as to understand the mechanisms giving rise to X-rays within PNe (Kastner et al. 2012). The ChanPlaNS survey mostly included high-excitation PNe and yielded X-ray detections from central star (point-like) and nebular (diffuse) X-ray sources.

We selected those ChanPlaNS PNe accessible to the IRAM $30 \mathrm{~m}$ telescope that had previously been detected ${ }^{1}$ in $\mathrm{CO}$ and/or $\mathrm{H}_{2}$. Physical properties of these nine PNe and their central stars are listed in Table 1. These properties include the morphology,

\footnotetext{
1 The lone exception, NGC 7008, was included in this survey due to its large X-ray luminosity.
}

Table 2. Radiative properties of $\mathrm{PNe}^{(1)}$.

\begin{tabular}{lcccc}
\hline \hline Name & $\begin{array}{c}L_{\mathrm{UV}} \\
\log \left(\mathrm{ergs} \mathrm{s}^{-1}\right)\end{array}$ & $\begin{array}{c}\text { X-ray } \\
\text { source }^{(2)}\end{array}$ & $\begin{array}{c}L_{\mathrm{X}} \\
\log \left(\mathrm{ergs} \mathrm{s}^{-1}\right)\end{array}$ & $\begin{array}{c}E_{\text {median }} \\
(\mathrm{keV})\end{array}$ \\
\hline BD+303639 & 37.23 & $\mathrm{D}$ & 32.20 & 0.74 \\
NGC 7027 & 37.47 & $\mathrm{D}$ & 32.11 & 0.97 \\
NGC 6445 & 36.57 & $\mathrm{P}$ & 30.10 & 1.04 \\
NGC 7008 & 36.72 & $\mathrm{P}$ & 29.48 & 0.85 \\
NGC 6720 & 36.18 & $\mathrm{~N}$ & $\leq 28.61$ & $\ldots$ \\
NGC 6853 & 36.09 & $\mathrm{P}$ & 29.15 & 0.18 \\
NGC 6772 & 36.01 & $\mathrm{~N}$ & $\leq 28.90$ & $\ldots$ \\
NGC 7293 & 35.55 & $\mathrm{P}$ & 29.94 & 0.89 \\
NGC 6781 & 36.15 & $\mathrm{~N}$ & $\leq 28.70$ & $\ldots$ \\
\hline
\end{tabular}

Notes. ${ }^{(1)}$ PN data obtained from Montez et al. (2015) unless otherwise specified. X-ray sources and median X-ray photon energies $\left(E_{\text {median }}\right)$ from Kastner et al. (2012), except NGC 7027 and BD+30 from Montez et al. (2015). Upper limit X-ray luminosities for non-detections provided by $\mathrm{R}$. Montez Jr. (unpublished). ${ }^{(2)} \mathrm{P}=$ point-like, $\mathrm{D}=$ diffuse, $\mathrm{N}=$ non-detection.

angular radius in arcsec, distance, average radius, age, and ionized mass of each PN, along with the spectral type, temperature, and mass of the CSPN as compiled from the literature. The ultraviolet luminosity, X-ray luminosity, median energy of emission, and X-ray source properties for each of the planetaries are listed in Table 2.

Data were collected for the nine listed PNe during an observing run on the IRAM $30 \mathrm{~m}$ telescope on Pico Veleta from 5th to 10th June, 2012. The positions for each targeted PN are listed in Table 3 and illustrated in Fig. 1. The observations were either centered on the PN central stars or the nebular rims; we obtained observations at two positions within NGC 6720, one toward the central star and one toward the rim. Frequencies in the regime of $88-236 \mathrm{GHz}$ were chosen to target key molecules for tracing chemistry. The molecular transitions detected during the course of the survey are listed in Table 4.

We used the Eight MIxer Receiver (EMIR), which is able to observe simultaneously in the 1 and $3 \mathrm{~mm}$ bands. Dual 
Table 3. Observed positions and integration times.

\begin{tabular}{lccc}
\hline \hline Source & $\alpha(\mathrm{J} 2000.0)$ & $\delta(\mathrm{J} 2000.0)$ & $\begin{array}{c}\text { Representative } \\
\text { integration time (h) }\end{array}$ \\
\hline BD+303639 & $19^{\mathrm{h}} 34^{\mathrm{m}} 45.23^{\mathrm{s}}$ & $30^{\circ} 30^{\prime} 58.90^{\prime \prime}$ & 5.76 \\
NGC 7027 & $21^{\mathrm{h}} 07^{\mathrm{m}} 1.59^{\mathrm{s}}$ & $42^{\circ} 14^{\prime} 10.20^{\prime \prime}$ & 2.56 \\
NGC 6445 & $17^{\mathrm{h}} 49^{\mathrm{m}} 15.21^{\mathrm{s}}$ & $-20^{\circ} 00^{\prime} 34.50^{\prime \prime}$ & 6.98 \\
NGC 7008 & $21^{\mathrm{h}} 00^{\mathrm{m}} 34.36^{\mathrm{s}}$ & $54^{\circ} 33^{\prime} 17.20^{\prime \prime}$ & 4.11 \\
NGC 6720 & $18^{\mathrm{h}} 53^{\mathrm{m}} 35.08^{\mathrm{s}}$ & $33^{\circ} 01^{\prime} 45.03^{\prime \prime}$ & 2.98 \\
NGC 6720 Rim & $18^{\mathrm{h}} 53^{\mathrm{m}} 31.90^{\mathrm{s}}$ & $33^{\circ} 01^{\prime} 25.00^{\prime \prime}$ & 2.98 \\
NGC 6853 & $19^{\mathrm{h}} 59^{\mathrm{m}} 36.38^{\mathrm{s}}$ & $22^{\circ} 43^{\prime} 15.80^{\prime \prime}$ & 11.01 \\
NGC 6772 & $19^{\mathrm{h}} 14^{\mathrm{m}} 36.37^{\mathrm{s}}$ & $-2^{\circ} 42^{\prime} 55.00^{\prime \prime}$ & 6.89 \\
NGC 7293 & $22^{\mathrm{h}} 29^{\mathrm{m}} 7.50^{\mathrm{s}}$ & $-20^{\circ} 48^{\prime} 58.60^{\prime \prime}$ & 7.79 \\
NGC 6781 & $19^{\mathrm{h}} 18^{\mathrm{m}} 27.42^{\mathrm{s}}$ & $6^{\circ} 31^{\prime} 29.30^{\prime \prime}$ & 5.43 \\
\hline
\end{tabular}

Notes. Integration times sampled from $\mathrm{HCN}, \mathrm{HCO}^{+}$, and $\mathrm{HNC}$ spectral region observations for each $\mathrm{PN}$.

polarization mode was used whenever each specific frequency setting made it possible, and the lines from the two polarizations were averaged after checking that their intensities were compatible. We recorded the data with the FTS200 backend, providing a resolution of $200 \mathrm{kHz}$, which translates into velocity resolutions of $\sim 0.5$ and $\sim 0.25 \mathrm{~km} \mathrm{~s}^{-1}$ in the 88 and $236 \mathrm{GHz}$ regimes, respectively, and a sufficient coverage of the lines listed above.

The beam sizes (Half Power Beam Widths) and beam efficiencies for the telescope ranged from $10.4^{\prime \prime}$ and 0.60 , respectively, for the $\mathrm{CO}^{+}$lines at $236 \mathrm{GHz}$ to $27.8^{\prime \prime}$ and 0.80 , respectively, for the HCN line at $88.6 \mathrm{GHz}$. Saturn and Mars were used to focus the telescope, and pointing errors were corrected to an accuracy of $\sim 3^{\prime \prime}$ by performing frequent measurements of nearby pointing calibrators (namely quasars such as QSO B1730130 and QSO J0854+2006, but also Neptune, the ionized region K3-50A, and the planetary nebula NGC 7027). The subreflector was wobbled with a throw of $120^{\prime \prime}$ every $2 \mathrm{~s}$ to provide stable and flat baselines. The data were calibrated in units of main-beam temperature, $T_{\mathrm{mb}}$ by frequent (15-20 min) calibration scans using the chopper wheel method. The $T_{\mathrm{mb}}$ values so obtained were then re-scaled using sources with stable, wellknown fluxes (CW Leo and NGC 7027). The estimated flux calibration accuracy is about $20 \%$.

Integration times varied from an hour for ${ }^{13} \mathrm{CO}$ lines to $6 \mathrm{~h}$ for $\mathrm{HCN}, \mathrm{HCO}^{+}$, and $\mathrm{HNC}$. Representative integration times are listed in Table 3. Weak lines were observed for significantly longer durations, up to $33 \mathrm{~h}$. System temperatures ranged between 102.4 and $445.9 \mathrm{~K}$, depending on the band and weather conditions.

Scan averaging and baseline subtraction of measurements were performed with the line analysis software available in CLASS $^{2}$ and through targeted inspection. Baseline oscillations were observed in some higher frequency spectra, but did not significantly affect measurements of individual molecular transitions.

\section{Results and analysis}

\subsection{Molecular line measurements}

Observations of the thirteen molecular lines detectable in the 88-236 GHz range provide new data for the nine $\mathrm{PNe}$ in our

\footnotetext{
2 https://www.iram.fr/IRAMFR/GILDAS/
}

survey, including the well observed NGC 7027. We identified and compiled a list of these molecular transitions, with lines of primary interest and their measured intensities summarized in Tables A.1 and A.2, respectively, for objects that have been well observed in the past and those for which this survey yielded a significant number of new detections. The tables list peak flux $\left(T_{\mathrm{mb}}\right)$ as defined by the main beam temperature, integrated line intensity ( $\int T_{\mathrm{mb}} \mathrm{d} V$ ), reported parameters for lines detected in past studies, and the $(1 \sigma)$ formal errors for each measurement. The values of temperature, intensity, and their errors were obtained from the Gaussian fitting procedure in CLASS.

\subsection{Survey overview}

We present the first detections of ${ }^{13} \mathrm{CO}(1-0,2-1), \mathrm{HCO}^{+}, \mathrm{CN}$, $\mathrm{HCN}$, and $\mathrm{HNC}$ in NGC 6445 (Sect. 3.3.6) as well as $\mathrm{HCO}^{+}$ in $\mathrm{BD}+30^{\circ} 3639$ (Sect. 3.3.5). Transitions of ${ }^{13} \mathrm{CO}(2-1), \mathrm{CN}$, $\mathrm{HCN}$, and $\mathrm{HNC}$ were also detected for the first time in NGC 6853 (Sect. 3.3.8) as well as ${ }^{13} \mathrm{CO}(2-1), \mathrm{CN}$, and $\mathrm{HCO}^{+}$in $\mathrm{NGC} 6772$ (Sect. 3.3.9). The individual PNe discussed in Sects. 3.3.1-3.3.4 have been the subject of previous radio molecular line surveys (Bachiller et al. 1997; Edwards et al. 2014; Zhang et al. 2008), and the present survey provided only a few new detections for these objects. Among the molecular ions surveyed, $\mathrm{HCO}^{+}$was detected in most of the PNe, including the aforementioned new detections in three objects. However, the only PN unambiguously detected in $\mathrm{CO}^{+}$and $\mathrm{N}_{2} \mathrm{H}^{+}$was NGC 7027 (Sect. 3.3.1), which was previously known to exhibit emission from both molecules (Hasegawa \& Kwok 2001; Zhang et al. 2008).

Figures 2-14 illustrate the emission lines detected in each object and compare their relative strengths. Across PNe with detected $\mathrm{CN}$, intensities of the strongest line scale more or less consistently with lesser $\mathrm{CN}$ hyperfine lines. With this in mind, we only list parameters for the brightest $\mathrm{CN}(1-0)$ hyperfine components in Tables A.1 and A.2. Hydrogen recombination lines were also detected in two PNe, NGC 7027 and $\mathrm{BD}+30^{\circ} 3639$. Both objects are very young and compact, with high ionized gas densities capable of producing strong atomic hydrogen emission. All CO isotopologue lines for NGC 7008 (Sect. 3.3.7) were also previously unobserved, however the detections obtained here are likely due to ISM gas along the line of sight to the PN.

We find line strengths for the PNe in our survey compare favorably with previous observations (e.g., Bachiller et al. 1997). Because many of the objects were observed at different positions, we limit direct comparisons to a pair of representative PNe. In NGC 7027, we find line ratios of ${ }^{12} \mathrm{CO} /{ }^{13} \mathrm{CO}$ and $\mathrm{HCO}^{+} /{ }^{13} \mathrm{CO}$ that are within $8 \%$ of those reported by Bachiller et al. (1997), while $\mathrm{CN} /{ }^{13} \mathrm{CO}$ is within $23 \%$. Similarly, we noted a difference of only $15 \%$ in the reported ${ }^{12} \mathrm{CO} /{ }^{13} \mathrm{CO}$ line ratio in NGC 6781, despite an offset of $50^{\prime \prime}$ between the positions observed within this PN in the two surveys.

\subsection{Individual planetary nebulae}

\subsubsection{NGC 7027}

The data obtained for NGC 7027 yield by far the richest molecular line spectrum among the objects included in this survey. This young, bright nebula has a notably hot CSPN $\left(T_{\star}=175000 \mathrm{~K}\right.$, Kastner et al. 2012; Frew 2008) and displays an abundance of atomic and molecular emission lines (Hasegawa \& Kwok 2001). With a dynamical age of only $700 \mathrm{yr}$ (Latter et al. 2000) and a high CSPN mass $\left(0.7 M_{\odot}\right)$, NGC 7027 is a rapidly evolving nebula (Zhang et al. 2008). 

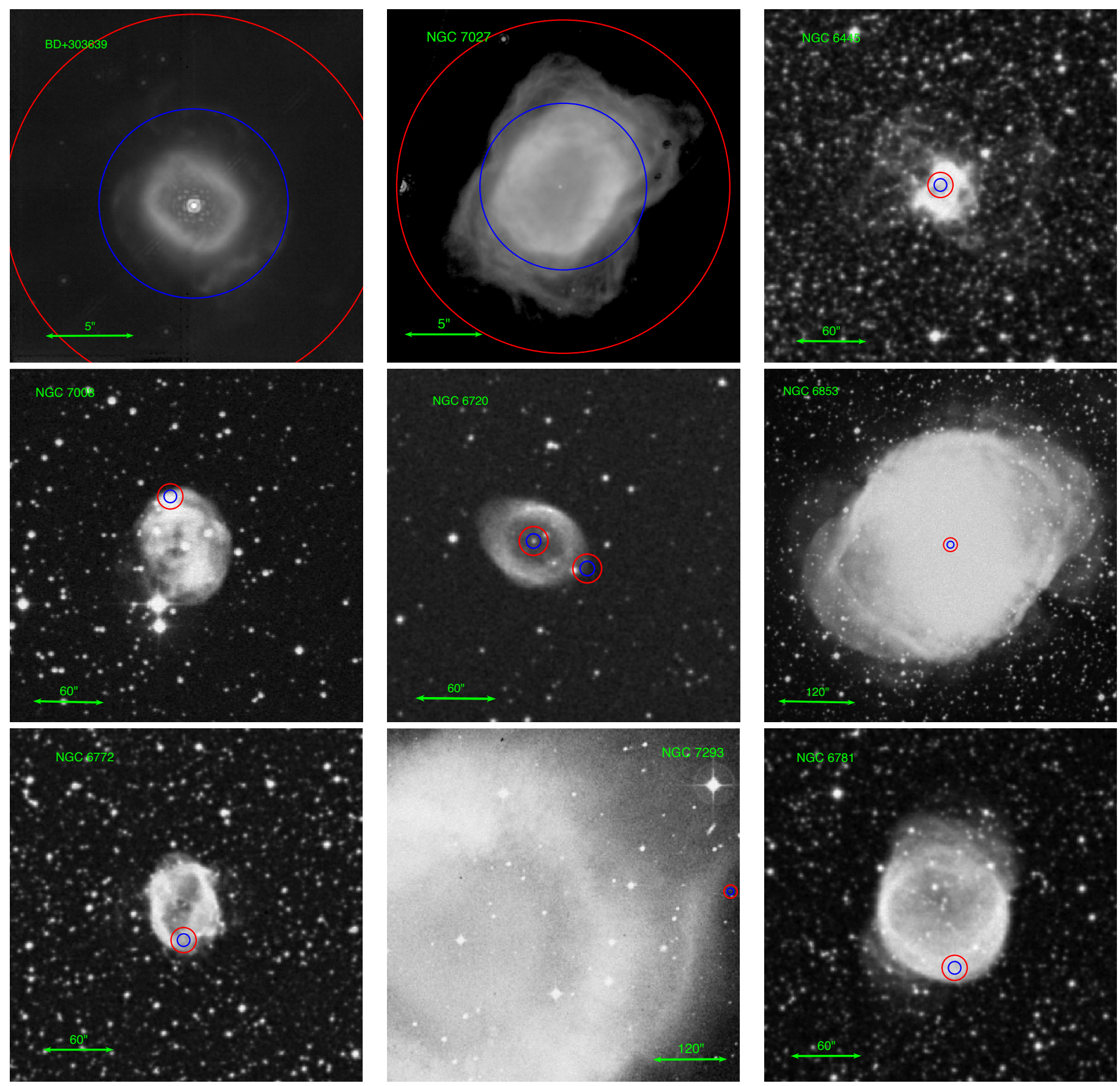

Fig. 1. POSS II red images for seven of the nine PNe observed in our study, with HST NICMOS images for BD+30 and NGC 7027. Circles illustrate beam diameter (half-power beam width) of the telescope and the position observed, where ${ }^{12} \mathrm{CO}(1-0)$ and ${ }^{12} \mathrm{CO}(2-1)$ were chosen to represent the range in beam size across frequency (red circle and blue circle, respectively). North is up, east is to the left in each frame.

The morphology of the HII region is that of a prolate ellipsoidal PN, with a distinct waist and symmetrical outflow lobes visible in the infrared and X-ray (Graham et al. 1993; Kastner et al. 2001). The clover-like symmetrical shell of molecular hydrogen forms a wispy and filamentary structure about the elongated shell of ionized gas (Latter et al. 2000; Cox et al. 2002). The $\mathrm{H}_{2}$ emission further traces out the thin PDR, separating the inner ionized gas from the expanding molecular envelope, which has been modeled spatio-kinematically by Santander-García et al. (2012) and determined to have a total molecular mass of roughly $1.3 M_{\odot}$. The presence of point-symmetric holes in the $\mathrm{H}_{2}$ region also indicates the presence of collimated outflows, a common phenomenon in young $\mathrm{PNe}$ (Cox et al. 2002).

All thirteen molecular transitions surveyed have been detected in NGC 7027 and their individual spectra are displayed in Figs. 2 and 3, which illustrate detections of neutral and ionized molecules, respectively. From the shapes of the spectral emission lines, several characteristics of this PN can be discerned. The distinct double-peaked structure found in $\mathrm{CO}$ isotopologues and $1 \mathrm{~mm}$ lines traces the molecular gas with high line-of-sight velocities. Because the molecular shell of NGC 7027 extends to 
Table 4. Molecular transitions detected.

\begin{tabular}{lrr}
\hline \hline Molecule & Transition & $v(\mathrm{GHz})$ \\
\hline $\mathrm{CO}$ & $J=1 \rightarrow 0$ & 115.2712018 \\
& $J=2 \rightarrow 1$ & 230.5380000 \\
${ }^{13} \mathrm{CO}$ & $J=1 \rightarrow 0$ & $110.2013543^{*}$ \\
& $J=2 \rightarrow 1$ & $220.3986842^{*}$ \\
$\mathrm{C}^{17} \mathrm{O}$ & $J=1 \rightarrow 0$ & $112.3587770^{*}$ \\
& $J=2 \rightarrow 1$ & $224.7141870^{*}$ \\
$\mathrm{HCN}$ & $J=1 \rightarrow 0$ & $88.6316022^{*}$ \\
$\mathrm{HNC}$ & $J=1 \rightarrow 0$ & 90.6635680 \\
$\mathrm{CN}$ & $N=1 \rightarrow 0$ & 113.1441573 \\
& & 113.1704915 \\
& & 113.1912787 \\
& $N=2 \rightarrow 1$ & $226.6595584^{*}$ \\
& & $226.8747813^{*}$ \\
$\mathrm{HCO}^{+}$ & $J=1 \rightarrow 0$ & 89.1885247 \\
$\mathrm{~N}_{2} \mathrm{H}^{+}$ & $J=1 \rightarrow 0$ & $93.1747000^{*}$ \\
$\mathrm{CO}^{+}$ & $J=2 \rightarrow 1$ & 235.7896410 \\
& & 236.0625530
\end{tabular}

Notes. Values of frequencies of hyperfine transitions obtained from the CDMS database. Line frequencies were measured in the laboratory by Müller et al. (2001), with updated catalog by Müller et al. (2005). Asterisks indicate frequencies representative of hyperfine transition complexes.

$>70^{\prime \prime}$ (Hasegawa \& Kwok 2001), the $\sim 10^{\prime \prime}$ beam of the $30 \mathrm{~m}$ at $1 \mathrm{~mm}$ misses much of the outer envelope gas emitting near the systemic velocity. In contrast, the larger beam size of our $3 \mathrm{~mm}$ observations more completely samples the entire shell of the nebula, resulting in a larger contribution from material expanding along the plane of the sky and, hence, flatter-topped line profiles. The same explanation applies to the difference between the line profiles measured here and those measured by Zhang et al. (2008) with the larger beam of the ARO $12 \mathrm{~m}$ telescope. Indeed, our CO isotopologue spectral line shapes are consistent with $30 \mathrm{~m}$ observations by Herpin et al. (2002).

The central velocity of NGC 7027 was estimated by averaging the widths of each profile and assuming symmetric expansion. The CO spectral line profiles show blueshifted and redshifted edges at 10.7 and $36.9 \mathrm{~km} \mathrm{~s}^{-1}$, respectively. We thereby obtain a systemic velocity of $23.8 \mathrm{~km} \mathrm{~s}^{-1}$ and an expansion velocity of $13.1 \mathrm{~km} \mathrm{~s}^{-1}$. These results are consistent with those of Graham et al. (1993), Cox et al. (2002), and Herpin et al. (2002), all of whom determined the systemic velocity of NGC 7027 to be approximately $25 \mathrm{~km} \mathrm{~s}^{-1}$ and found molecular outflow velocities of $15 \mathrm{~km} \mathrm{~s}^{-1}$. The nitrogen-bearing molecules, however, present a slightly faster expanding shell, with blueshifted and redshifted outflow velocities of 5 and $40 \mathrm{~km} \mathrm{~s}^{-1}$, respectively. The velocity difference may be attributed to the N-bearing molecules being more abundant in the collimated, higher-velocity outflows present in NGC 7027.

We also observe evidence of self-absorption in the $\mathrm{HCO}^{+}$ spectrum and possibly in CO (1-0); on the blueshifted side of the $\mathrm{HCO}^{+}$emission line, there appear absorption features at $\sim 0.8,3.2$, and $11.0 \mathrm{~km} \mathrm{~s}^{-1}$ that dip below the averaged continuum, which has been baseline-subtracted (Fig. 4). We believe these are circumstellar features and not interstellar as there is no
NGC 7027

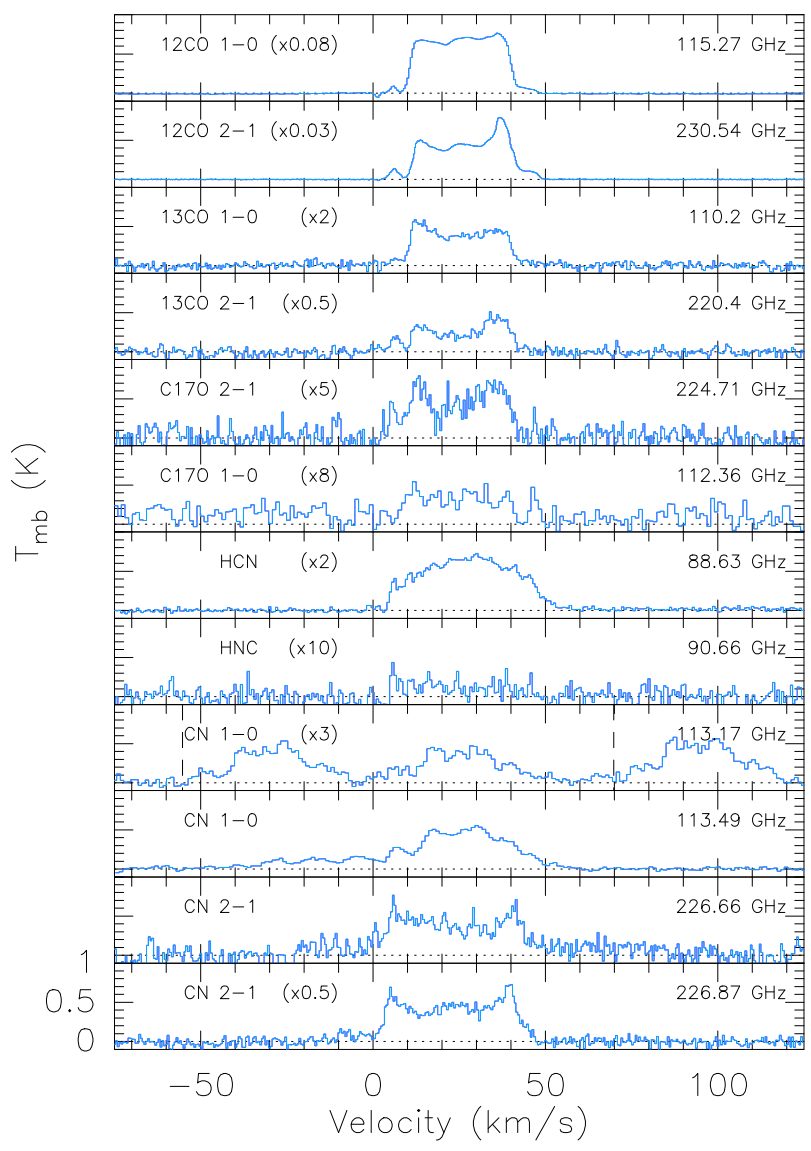

Fig. 2. Spectra for transitions of neutral molecules detected in the survey data for NGC 7027. Individual spectra have been scaled to facilitate comparison, and vertical dashed lines have been added to indicate the systemic velocity positions for the hyperfine $\mathrm{CN}$ lines. The $x$-axis indicates velocity with respect to the local standard of rest $\left(v_{\mathrm{LSR}}\right)$ and the $y$-axis is main-beam antenna temperature $(\mathrm{K})$.

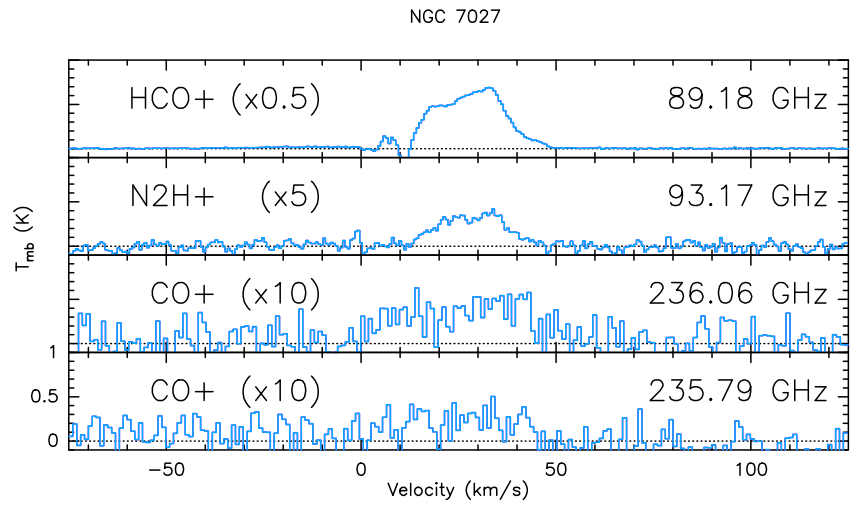

Fig. 3. As in Fig. 2, but for transitions of molecular ions detected in the survey data for NGC 7027.

observed extended emission in wide $\mathrm{CO}$ maps or self-absorption in higher CO J lines (Santander-García et al. 2012). Beyond the absorption features, an extended blueshifted wing is apparent within the $\mathrm{HCO}^{+}$and $\mathrm{CO}(1-0)$ spectra (Fig. 4). Extended ${ }^{12} \mathrm{CO}$ wings were first detected in NGC 7027 by Bujarrabal et al. (2001). While the $\mathrm{CO}$ wing is less extensive, the $\mathrm{HCO}^{+}$wing extends to $\sim-40 \mathrm{~km} \mathrm{~s}^{-1}$, indicating the presence of a molecular 


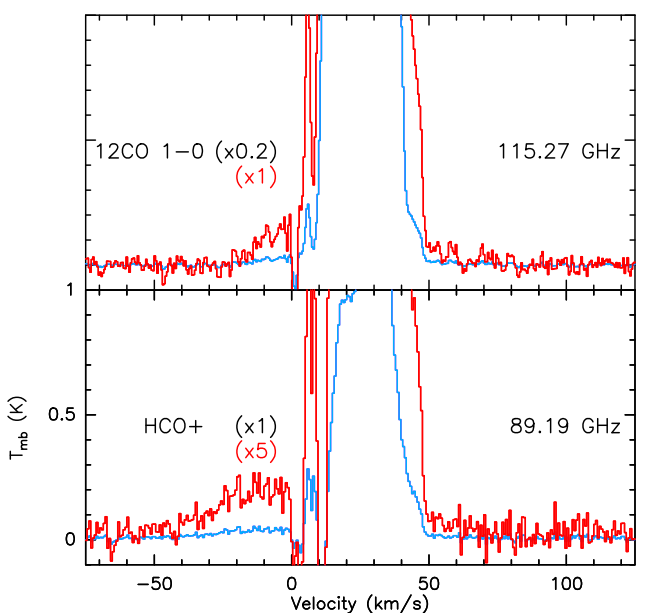

Fig. 4. Expanded spectra of $\mathrm{CO}$ and $\mathrm{HCO}^{+}$in $\mathrm{NGC} 7027$, illustrating the presence of multiple absorption features and an extended blue wing.

outflow with velocity $\sim 65 \mathrm{~km} \mathrm{~s}^{-1}$, i.e., nearly 5 times that of the overall molecular expansion velocity. This velocity is strictly a lower limit, dependent on the outflow inclination. This suggests that low excitation, approaching regions of the outer molecular envelope are absorbing the line and continuum emission from molecules of high abundance in the inner regions of the PN.

We confirm the detection of the ion $\mathrm{N}_{2} \mathrm{H}^{+}$made by Zhang et al. (2008). Formation of $\mathrm{N}_{2} \mathrm{H}^{+}$could be a result of the strong presence of X-rays from the CSPN, or due to shocks in the pPN phase.

While the double-peaked structure that is characteristic of an expanding envelope of molecular gas can be seen in most of the emission lines observed, it is notably absent in $\mathrm{HCN}$, due to its hyperfine splitting at closely spaced frequencies $(88.630,88.631$, and $88.634 \mathrm{GHz}$ ). Line broadening from expansion blends these hyperfine features. Close hyperfine lines may also be blended and thus unresolved in ${ }^{13} \mathrm{CO}, \mathrm{C}^{17} \mathrm{O}$, and $\mathrm{N}_{2} \mathrm{H}^{+}$. $\mathrm{CN}$ displays hyperfine structure as well (Table 4 ), but at generally separable frequencies for both the $N=2-1$ and $N=1-0$ transitions. Two of the three hyperfine components of $\mathrm{CO}^{+}$are detected (Fig. 3).

Further analysis of the spectra revealed detections of hydrogen recombination lines at $92.0(\mathrm{H} 41 \alpha)$ and $222(\mathrm{H} 38 \beta) \mathrm{GHz}$ (Fig. 5). $\mathrm{H} 41 \alpha$ has a measured peak intensity of $0.278 \mathrm{~K}$ and integrated flux of $4.12 \mathrm{~K} \mathrm{~km} \mathrm{~s}^{-1}$, and was previously identified by Zhang et al. (2008). H38 $\beta$ has a peak intensity of $0.096 \mathrm{~K}$ and an integrated flux of $2.63 \mathrm{~K} \mathrm{~km} \mathrm{~s}^{-1}$.

\subsubsection{NGC 6720}

Commonly known as the Ring Nebula, NGC 6720 (M 57) has been well studied in molecular gas (Bachiller et al. 1997; Edwards et al. 2014). Its dynamical age is roughly $7000 \mathrm{yr}$ (O'Dell et al. 2007) and it does not have detectable X-ray emission (Kastner et al. 2012). We observed two positions within NGC 6720, one toward the CSPN, and one just outside the optically bright nebula (Fig. 1). Emission lines for these regions are displayed in Figs. 6 and 7, respectively. Given the extensive CO survey of NGC 6720 performed by Bachiller et al. (1989a), we chose not to cover higher frequency wavebands (including three of the $\mathrm{CO}$ isotopologues) in our survey observations, and as such only six molecular transitions were detected.
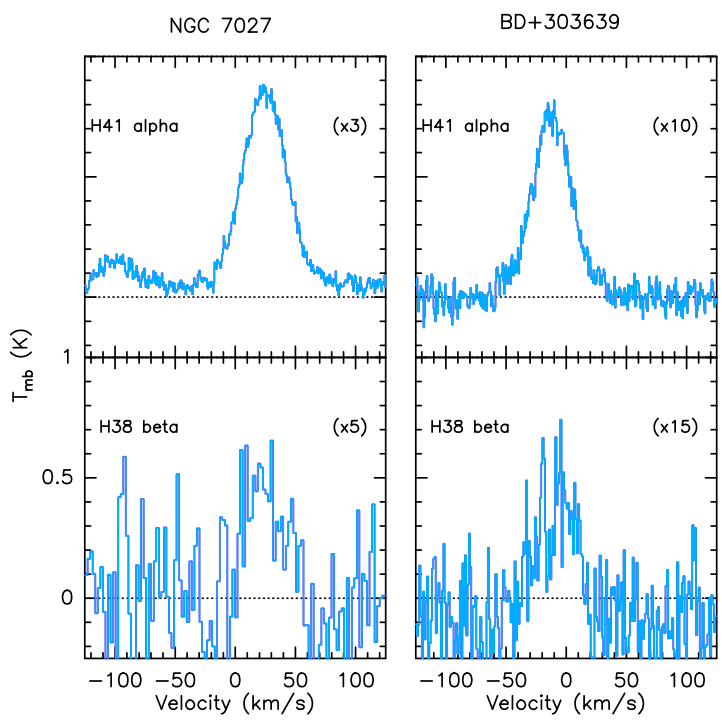

Fig. 5. Hydrogen recombination lines detected in two of the surveyed PNe, NGC 7027 and BD+303639.

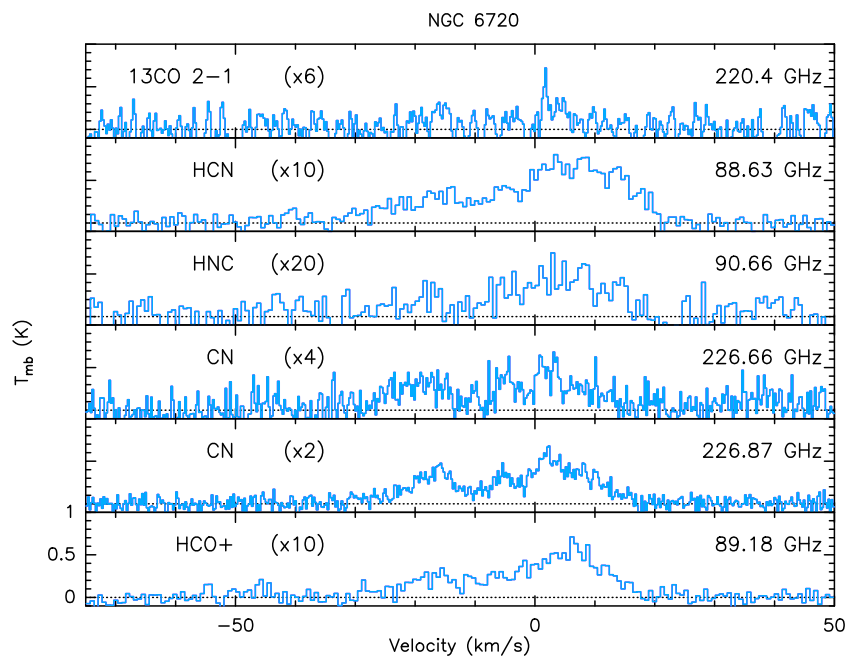

Fig. 6. As in Fig. 2, molecular transitions detected in the survey data obtained toward the central star of NGC 6720.

The CSPN pointing (Fig. 6) encompasses molecular gas associated with both the approaching and receding layers of the molecular shell, and as a result this pointing yields broad, double-peaked line profiles. We measure blueshifted and redshifted velocity components of -16.2 and $4.6 \mathrm{~km} \mathrm{~s}^{-1}$, respectively, at the central star of NGC 6720. These values agree well with the CO measurements of Edwards et al. (2014), though they also detect a redshifted component at $15.7 \pm 2.6 \mathrm{~km} \mathrm{~s}^{-1}$.

The spectra for the position offset from the CSPN yield line profiles that are dominated by emission near the systemic velocity of the PN, consistent with the expectation that the velocity of the bulk of the gas along the nebular rim is perpendicular to the line of sight. The extended tail toward the blueshifted side of the nebula indicates that the emission is dominated by the forward-directed side of the molecular region. At this rim position, emission peaks appear at $-6.7 \mathrm{~km} \mathrm{~s}^{-1}$ and $-0.8 \mathrm{~km} \mathrm{~s}^{-1}$. Another peak was identified at $15.3 \mathrm{~km} \mathrm{~s}^{-1}$, but only in $\mathrm{H}$-bearing molecules. This spectral feature in the Rim position corresponds to the receding emission component detected in molecular spectra obtained by Edwards et al. (2014). Although we did not 


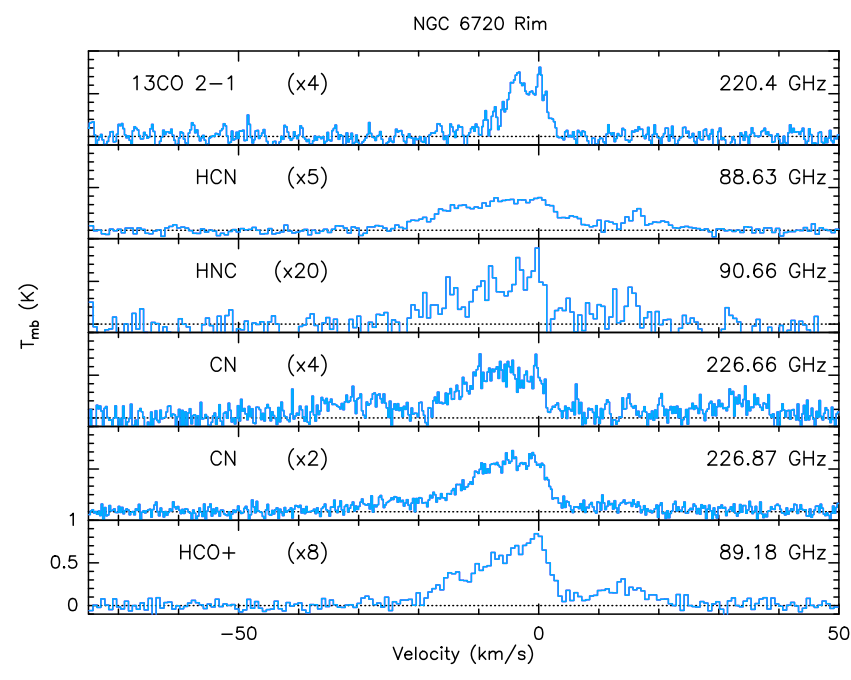

Fig. 7. As in Fig. 2, molecular transitions detected in the survey data obtained toward the rim of NGC 6720, referred to throughout this paper as NGC 6720 Rim.

NGC 7293

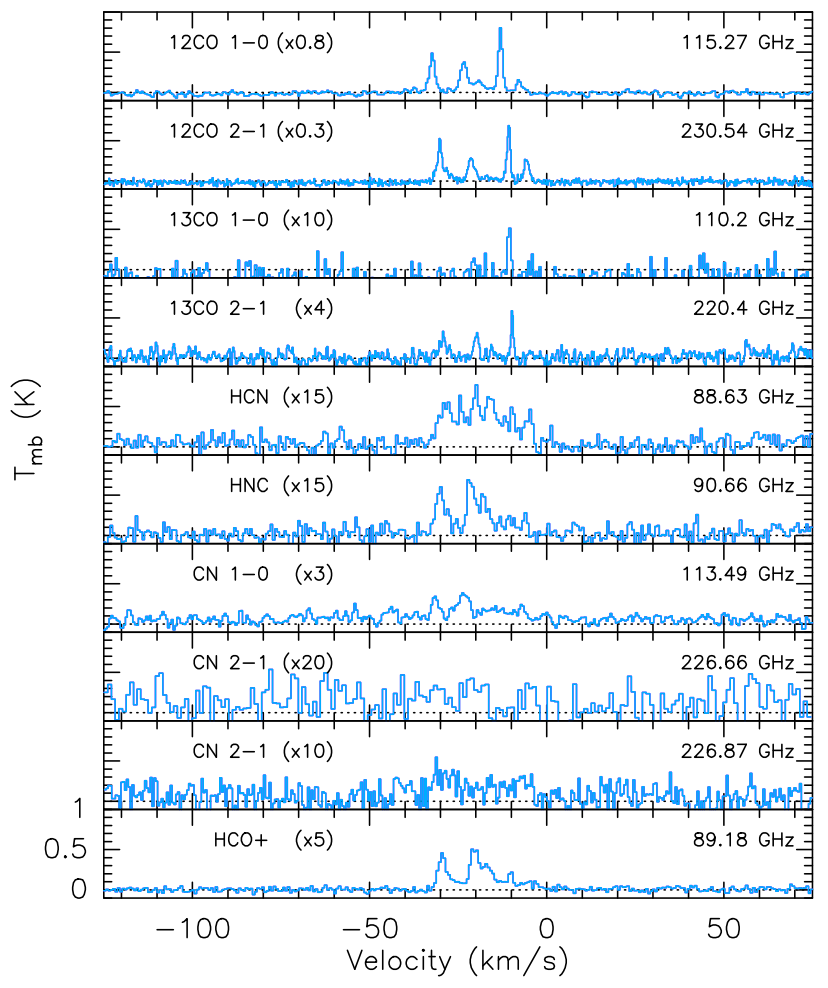

Fig. 8. As in Fig. 2, molecular transitions detected in the survey data for NGC 7293.

observe CO (2-1) in NGC 6720, the observed profiles match the structure seen by Bachiller et al. (1989a) in their $\left(\Delta \alpha=-40^{\prime \prime}\right.$, $\left.\Delta \gamma=-20^{\prime \prime}\right)$ position. Further, the average systemic velocity of NGC 6720 across both positions (approximately $-0.8 \mathrm{~km} \mathrm{~s}^{-1}$ ) agrees well with the measurements of Bachiller et al. (1997).

\subsubsection{NGC 7293}

At a distance of only $0.20 \mathrm{kpc}$ (Gaia Collaboration 2018) and a radius of $0.46 \mathrm{pc}$, the Helix Nebula (NGC 7293) represents the largest and nearest $\mathrm{PN}$ in this study. It is a relatively evolved object with a dynamical age of 12000 yr (Schmidt \& Ziurys 2017a), and contains a hard, point-like X-ray source (Guerrero et al. 2001). Early radio molecular line observations were carried out by Huggins et al. (1992) and Bachiller et al. (1997), with many additional studies performed across the expansive molecular emission region of the PN (Young et al. 1999; Zack \& Ziurys 2013; Zeigler et al. 2013; Schmidt \& Ziurys 2017a; Schmidt et al. 2018).

Our observations of NGC 7293 were performed toward the edge of the molecular envelope (Fig. 1). While all the molecular transitions studied in this paper have previously been detected in this PN, this work adds integrated flux values for these lines in a previously unobserved region of the object. As in previous single-dish mapping studies, there is significant structure present in the spectra (Fig. 8). In particular, four distinct peaks appear in the ${ }^{12} \mathrm{CO}(1-0,2-1)$ transitions. This complex line profile can be attributed to multiple, distinct molecular knots along the line of sight sampled by the $30 \mathrm{~m}$ beam. Due to the large angular diameter of NGC 7293, the IRAM telescope beam measured only a small portion of the extensive molecular region. As such, it is only possible to make a comparison with a nearby region $\left(\Delta \alpha=-435^{\prime \prime}, \Delta \gamma=75^{\prime \prime}\right)$, as observed by Zack \& Ziurys (2013), Schmidt \& Ziurys (2017a), and Schmidt et al. (2018). Their spectra revealed three CO velocity components at $-11,-21$, and $-29 \mathrm{~km} \mathrm{~s}^{-1}$ and $-13,-21,-29 \mathrm{~km} \mathrm{~s}^{-1}$ in $\mathrm{HNC}$ and $\mathrm{HCN}$, respectively. This lines up well with our spectra, in which strong velocity components are observed at roughly $-13,-23$, and $-32 \mathrm{~km} \mathrm{~s}^{-1}$, with two other potential features at -8 and $-19 \mathrm{~km} \mathrm{~s}^{-1}$. Observations by Schmidt et al. (2018) of CN at the nearby position $(-435,75)$, as well as seven other positions across the Helix, detected hyperfine structure indicative of optically thin emission; our data are too noisy to confirm these results.

\subsubsection{NGC 6781}

NGC 6781 is a highly evolved PN with an estimated dynamical age of at least $20000 \mathrm{yr}$ (Ueta et al. 2014), making it the oldest object included in our survey. As one of our three surveyed PNe not detected in X-rays, NGC 6781 acts as a control for any potential X-ray-induced chemistry effects.

Radio molecular line observations of NGC 6781 were previously carried out by Bachiller et al. (1997). New observations of the PN presented here probe a different region of the expanding shell. NGC 6781 was detected in all molecular transitions except $\mathrm{CO}^{+}$and $\mathrm{N}_{2} \mathrm{H}^{+}$(Fig. 9). The molecular line profiles of NGC 6781 display complex structures. At least four distinct velocity components were detected across the lines. Blueshifted and redshifted components at -0.3 and $29.4 \mathrm{~km} \mathrm{~s}^{-1}$ respectively were detected for all molecular transitions. This suggests a systemic velocity of $14.6 \mathrm{~km} \mathrm{~s}^{-1}$. A central component at $14.7 \mathrm{~km} \mathrm{~s}^{-1}$ was also ubiquitously detected, with a fourth line at $21.4 \mathrm{~km} \mathrm{~s}^{-1}$ identified in most of the transitions. These velocity profiles generally match up with those presented in Bachiller et al. (1997), who found velocity extremes of $\mathrm{CO}$ from -5 to $39 \mathrm{~km} \mathrm{~s}^{-1}$.

The strong, narrow absorption component located at $\sim 6.5 \mathrm{~km} \mathrm{~s}^{-1}$ in the $\mathrm{CO}$ line profiles is present across all detected transitions and is likely due to the presence of molecular cloud emission in the off-source (background sky) reference position.

\subsection{5. $\mathrm{BD}+30^{\circ} 3639$}

Also known as Campbell's Star, BD+30³639 (hereafter $\mathrm{BD}+30$ ), one of the youngest and most compact $\mathrm{PN}$ in our 
NGC 6781

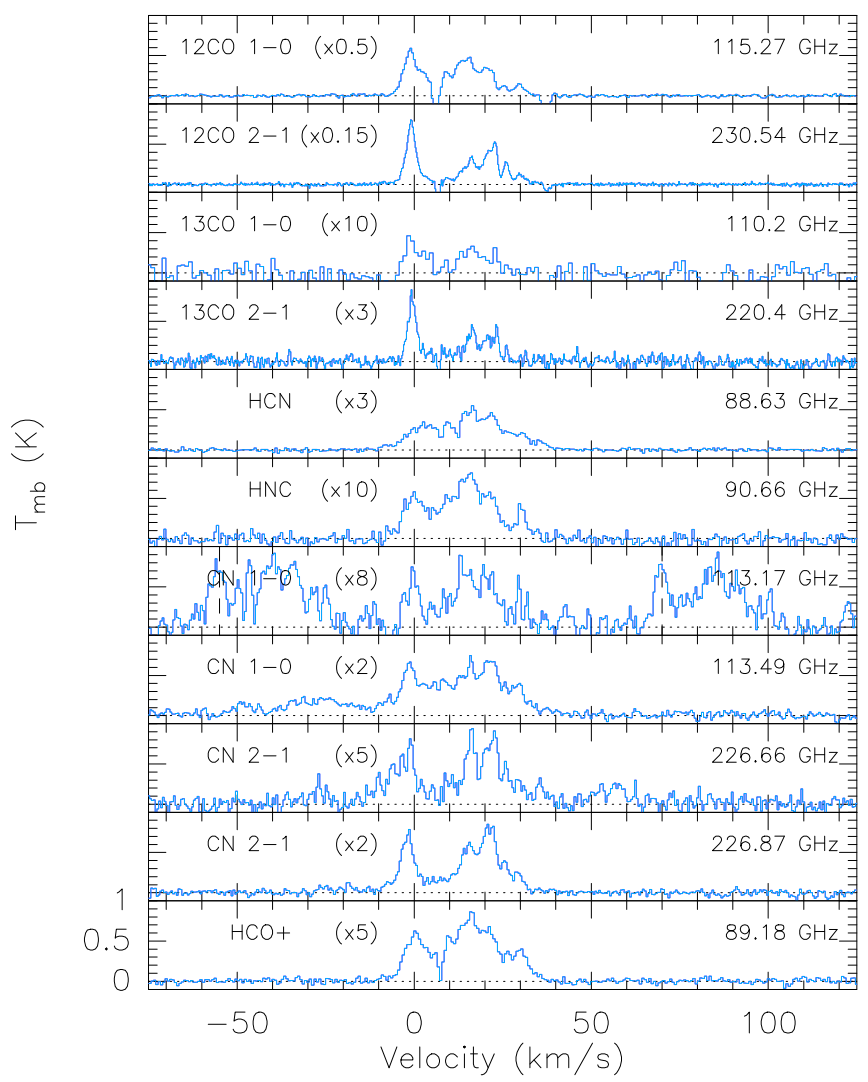

Fig. 9. As in Fig. 2, molecular transitions detected in the survey data for NGC 6781 . Vertical dashed lines indicate the systemic velocity positions for the hyperfine $\mathrm{CN}$ lines.

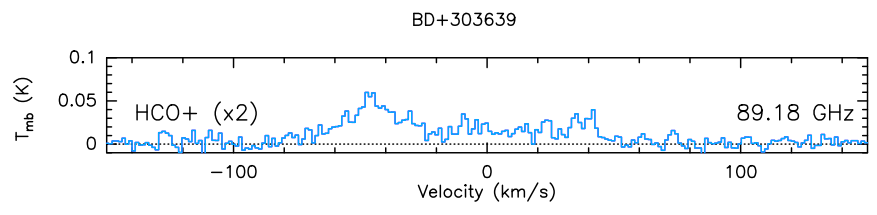

Fig. 10. As in Fig. 2, the molecular transition detected in the survey data for $\mathrm{BD}+30^{\circ} 3639$.

survey, has only just begun ionizing its envelope of ejected material within the past $1000 \mathrm{yr}$ ( $\mathrm{Li}$ et al. 2002). The WolfRayet-type central star (WC9) represents the coolest core of the $\mathrm{PNe}$ discussed in this paper. Nevertheless, BD+30 is a luminous object; at $10^{37} \mathrm{ergs} \mathrm{s}^{-1}$, its CSPN UV luminosity is rivaled only by NGC 7027. It is also among the brightest diffuse PN X-ray sources (Kastner et al. 2012), and has been studied extensively across the electromagnetic spectrum (Freeman \& Kastner 2016). Still, it remains poorly characterized in molecular emission.

The structure of BD+30 is that of an elliptical nebula, with asymmetric $\mathrm{CO}$ bullets along possibly precessing jets (Bachiller et al. 1991; Akras \& Steffen 2011; Gussie \& Taylor 1995, and references therein). These misaligned jets may also be responsible for a hot bubble of X-ray-emitting material bounded by the ionized inner shell and outer dusty region (Akras \& Steffen 2011; Freeman \& Kastner 2016). An extensive multi-wavelength 3D structure modeling study was performed by Freeman \& Kastner (2016).

In our survey data for $\mathrm{BD}+30$, only $\mathrm{HCO}^{+}$was detected (Fig. 10). BD+30 subtends a diameter of $4^{\prime \prime}$ (Frew 2008) and its CO emission is compact (Bachiller et al. 1991); hence, beam dilution may have a deleterious effect on $\mathrm{CO}$ and other molecules surveyed. The $\mathrm{HCO}^{+}$line profile displays a fairly broad structure, with two distinct peaks. We find the expansion velocity of these regions within $\mathrm{BD}+30$ to be -46.0 and $38.4 \mathrm{~km} \mathrm{~s}^{-1}$ respectively. Compared with the observations by Bachiller et al. (1991), who determined $\mathrm{CO}$ bullet velocities of -63 and $41 \mathrm{~km} \mathrm{~s}^{-1}$, a puzzling discrepancy emerges. The redshifted $\mathrm{HCO}^{+}$component matches the $\mathrm{CO}$ velocity, but the approaching velocity component differs by $\sim 17 \mathrm{~km} \mathrm{~s}^{-1}$, a significant offset. The velocity extension of the $\mathrm{HCO}^{+}$emission suggests that the molecular ion is not contained solely within the CO bullets but spread across the PN. Future followup with interferometer observations will be necessary to confirm this inference.

As in the case of NGC 7027, examination of the spectra revealed the presence of strong $\mathrm{H} 41 \alpha$ and $\mathrm{H} 38 \beta$ emission lines (Fig. 5), with peak intensities $0.076,0.033 \mathrm{~K}$ and integrated flux values of $1.02,1.02 \mathrm{~K} \mathrm{~km} \mathrm{~s}^{-1}$, respectively. These $\mathrm{H}$ recombination lines will not be further discussed in this paper, but they are consistent with the presence of a large mass of ionized hydrogen in $\mathrm{BD}+30$.

\subsubsection{NGC 6445}

NGC 6445, or the Little Gem, is the most distant PN in this survey, at $1.38 \mathrm{kpc}$ (Frew et al. 2016). It appears as a bipolar nebula of dynamical age roughly 5000 yr (Phillips 1984) and its CSPN has a temperature of $T_{\star}=170000 \mathrm{~K}$. NGC 6445 is at a comparable effective temperature and CSPN mass to NGC 7027, though it has far lower CSPN luminosity and larger nebula as a consequence of its more evolved state. The ChanPlaNs survey established that NGC 6445 harbors a compact $\mathrm{X}$-ray source of uncertain physical origin, with coronal emission from a binary companion being a likely source (Montez et al. 2015). The $\mathrm{CO}$ isotopologues along with $\mathrm{OH}$ and $\mathrm{OH}^{+}$ were the only molecules previously detected in NGC 6445 (Huggins \& Healy 1989; Sun \& Sun 2000; Aleman et al. 2014).

Here, we present the detection of five new molecular species: ${ }^{13} \mathrm{CO}, \mathrm{HCO}^{+}, \mathrm{CN}, \mathrm{HCN}$, and $\mathrm{HNC}$. The shapes of these emission lines vary, with the nitrogen-bearing molecules displaying the broadest profiles (Fig. 11). For those lines that are welldetected, double peaked structure is apparent and provides an estimated systemic velocity for the PN of $18.6 \mathrm{~km} \mathrm{~s}^{-1}$ and outflow velocity of $22.0 \mathrm{~km} \mathrm{~s}^{-1}$. The lack of a blueshifted edge in the $\mathrm{CO}$ lines suggests weaker $\mathrm{CO}$ emission from the approaching side of NGC 6445. Nevertheless, these velocity measurements are comparable with those of Sun \& Sun (2000) who measured a CO (1-0) systemic velocity of $20 \mathrm{~km} \mathrm{~s}^{-1}$ and an expansion velocity of $33 \mathrm{~km} \mathrm{~s}^{-1}$. A feature near the systemic velocity at $20.4 \mathrm{~km} \mathrm{~s}^{-1}$ can also be seen in the $\mathrm{HCO}^{+}$and $\mathrm{CN}$ lines. The ${ }^{12} \mathrm{CO}(1-0)$ transition also shows a dip in its emission line spectrum at $115.276 \mathrm{GHz}$ that is likely the result of over-subtracted ISM molecular emission along the line of sight. There is some hint of $\mathrm{N}_{2} \mathrm{H}^{+}$emission at $\sim 0 \mathrm{~km} \mathrm{~s}^{-1}$, within the velocity range of the other well-detected emission lines, but given the absence of emission at $\sim 40 \mathrm{~km} \mathrm{~s}^{-1}$ - where all the other lines peak - we do not regard this as a detection of $\mathrm{N}_{2} \mathrm{H}^{+}$.

\subsubsection{NGC 7008}

NGC 7008 is a relatively young nebula, with a dynamical age of $5700 \mathrm{yr}$ (Schmidt \& Ziurys 2016). Despite not being previously detected in molecular line emission, it was included 
NGC 6445

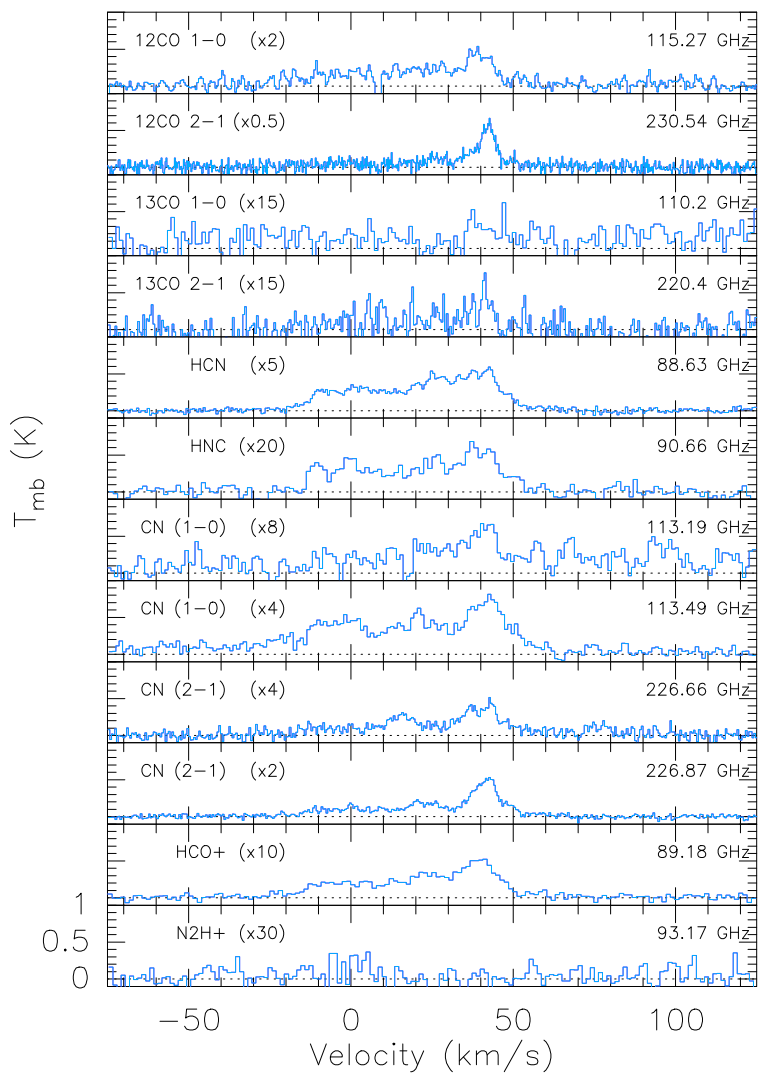

Fig. 11. As in Fig. 2, molecular transitions detected and tentatively detected in the survey data for NGC 6445.

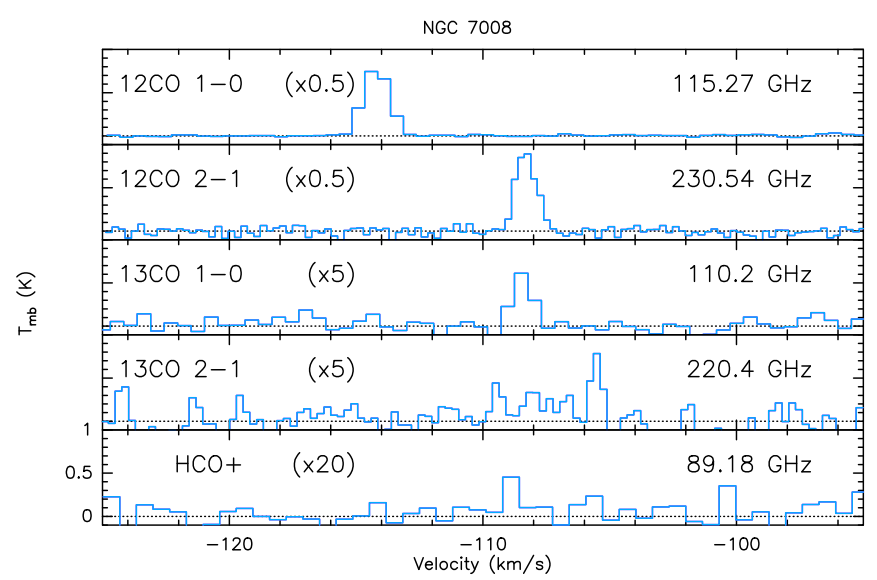

Fig. 12. As in Fig. 2, molecular transitions tentatively detected in the survey data for NGC 7008 .

in this survey for its strong X-ray emission; as in the case of NGC 6445, the CSPN is detected as a point-like X-ray source with an unconstrained emission origin (Montez et al. 2015). Previous molecular line measurements have been restricted to upper limits placed on $\mathrm{HCO}^{+}$and $\mathrm{HCN}$ (Schmidt \& Ziurys 2016).

Observations were made along the northern edge of the molecular shell of NGC 7008 (Fig. 1), yielding detections of narrow $\mathrm{CO}$ lines. Given the narrow widths and varying systemic velocities of the detected lines (Fig. 12), we believe these emission lines of $\mathrm{CO}$ isotopologues originate from the ISM rather than from the nebula. Followup observations of NGC 7008

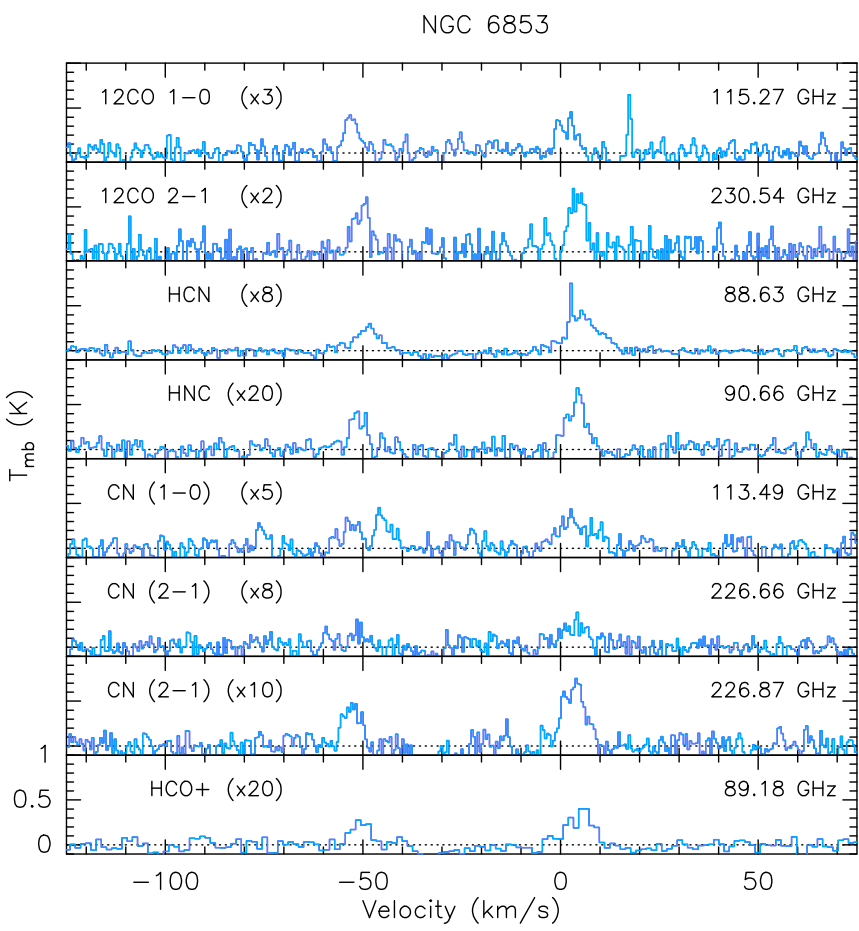

Fig. 13. As in Fig. 2, molecular transitions detected in the survey data for NGC 6853 .

are necessary to establish the origin of $\mathrm{CO}$ emission towards the PN.

\subsubsection{NGC 6853}

NGC 6853, also known as M 27 or the Dumbbell Nebula, is a large PN at $0.74 \mathrm{pc}$ in diameter that subtends $7500 \operatorname{arcsec}^{2}$ on the sky. In near-IR $\mathrm{H}_{2}$ emission, the Dumbbell Nebula consists of dense knots and "streamers" that appear to radiate out from the CSPN (Kastner et al. 1996; Salama et al. 2000). Numerous atomic lines in the optical such as [O I] and [N II] further trace out the eponymous "dumbbell" shape that reflects the PN's bipolar structure (Edwards et al. 2014). In the ChanPlaNs survey, NGC 6853 was detected as a point-like source of X-ray emission, with intensity and low median energy as expected from photospheric emission due to the hot $\left(T_{\star}=135000 \mathrm{~K}\right) \mathrm{CSPN}$, given its small distance of $0.43 \mathrm{kpc}$ (Frew et al. 2016).

CO was first detected in NGC 6853 by Huggins et al. (1996), who identified a complex filamentary structure much like that seen in $\mathrm{H}_{2}$. Further observations were carried out by Salama et al. (2000) and more recently by Edwards et al. (2014). In the latter, the study focused on a position offset from the CSPN $\left(\Delta \alpha=-68^{\prime \prime}, \Delta \gamma=-63^{\prime \prime}\right)$, where transitions of $\mathrm{CO}, \mathrm{HCO}^{+}$, and $\mathrm{CS}$ were identified. The region of the nebula targeted here is centered on the CSPN (Fig. 1). $\mathrm{H}_{2}$ imaging revealed this line-ofsight intersects a large column of clumpy, molecular gas (Kastner et al. 1996).

In our $30 \mathrm{~m}$ spectra, a well defined double-peaked structure is observed, with blueshifted and redshifted components of -50.3 and $4.9 \mathrm{~km} \mathrm{~s}^{-1}$, respectively (Fig. 13). When compared with the velocities of emission line components detected by Edwards et al. (2014) towards the edge of the PN $\left(-35\right.$ and $-5 \mathrm{~km} \mathrm{~s}^{-1}$, respectively) the velocity components we detect hint at the $3 \mathrm{D}$ structure of the PN. The measured velocity components indicate a systemic velocity of $-22.7 \mathrm{~km} \mathrm{~s}^{-1}$ and expansion velocity of $27.6 \mathrm{~km} \mathrm{~s}^{-1}$ for the expanding molecular shell of NGC 6853. 


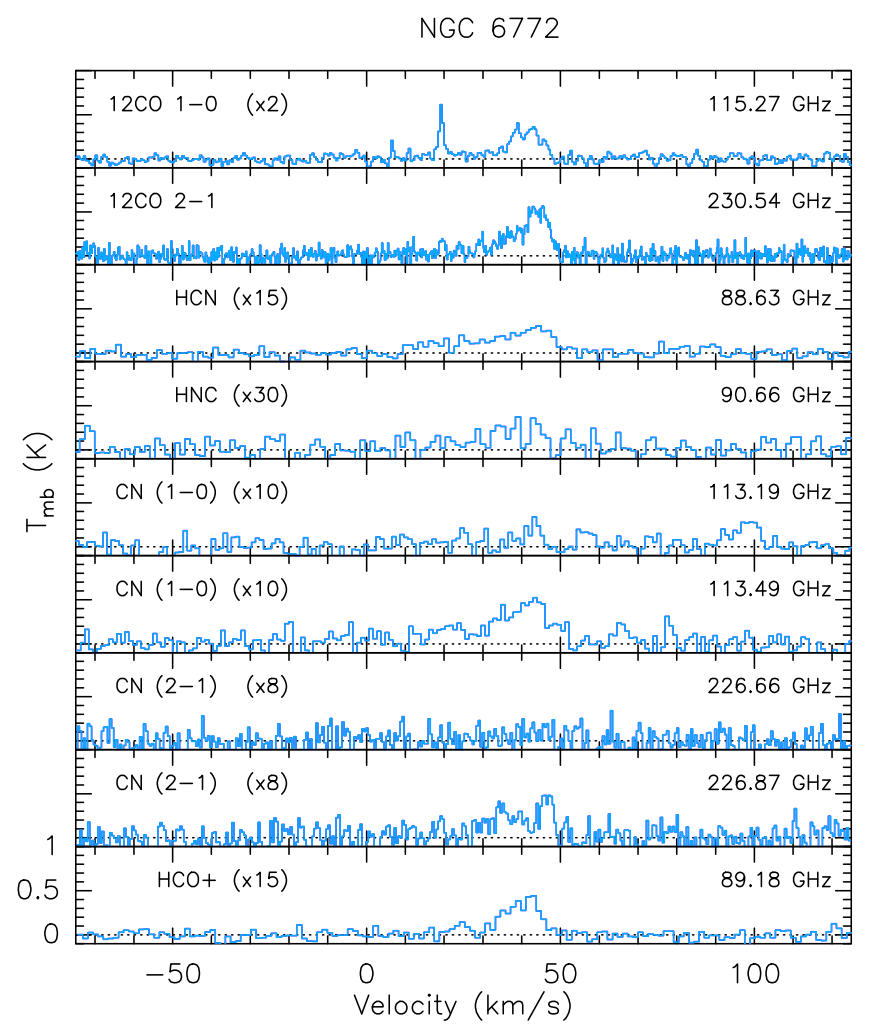

Fig. 14. As in Fig. 2, molecular transitions detected in the survey data for NGC 6772.

\subsubsection{NGC 6772}

One of the older objects in this sample, with a dynamical age of $11000 \mathrm{yr}$ (Ali et al. 2012), the morphology of NGC 6772 is that of an elliptical shell with apparent distortion along the NE to SW axis. This structure may be a result of interactions between the expanding shell and the ISM (Schmidt \& Ziurys 2016). NGC 6772 is one of three PNe in this paper not detected in X-rays in the ChanPlaNS survey (Kastner et al. 2012). First detected in CO (1-0) by Zuckerman et al. (1990), Schmidt \& Ziurys (2016) have more recently identified emission lines of CO (2-1) and HCN.

Our molecular line data were collected along the southern edge of the envelope (Fig. 1). The $30 \mathrm{~m}$ spectra yield detections of both previously detected $\mathrm{CO}$ transitions, as well as ${ }^{13} \mathrm{CO}(2-1), \mathrm{CN}$, and $\mathrm{HCO}^{+}$(Fig. 14). The broad peaks of the spectral line profiles terminate at a redshifted velocity of approximately $44.2 \mathrm{~km} \mathrm{~s}^{-1}$. This is consistent with the value determined by Schmidt \& Ziurys (2016). Only ${ }^{12} \mathrm{CO}(1-0)$ displays a narrow blueshifted peak at roughly $19.3 \mathrm{~km} \mathrm{~s}^{-1}$, as well as an apparent narrow feature near $0 \mathrm{~km} \mathrm{~s}^{-1}$ that was also identified by Schmidt \& Ziurys (2016). A possible explanation for this narrow feature is interstellar gas along the line of sight to NGC 6772.

\section{Discussion}

\subsection{HNC/HCN ratio}

The HNC/HCN abundance ratio has been studied for the insight it provides into the physical conditions within the photodissociation regions of cold molecular clouds. Activation energy barriers and the existence of a critical temperature for the conversion between $\mathrm{HNC}$ and $\mathrm{HCN}$, both formed predominantly by dissociative recombination of $\mathrm{HCNH}^{+}$, have long been discussed
(Schilke et al. 1992; Bachiller et al. 1997; Schmidt \& Ziurys 2017a). For semi-ionized gas near the PDR boundary, heightened temperatures should favor production and survival of $\mathrm{HCN}$, decreasing the $\mathrm{HNC} / \mathrm{HCN}$ ratio. The lack of convergence on the fundamental mechanisms and parameters that establish the ratio is apparent in the current literature, however (Graninger et al. 2014, and references therein).

Schilke et al. (1992) studied $\mathrm{HNC} / \mathrm{HCN}$ as a diagnostic of local gas heating in the Orion Molecular Cloud (OMC-1), and in particular, explored the temperature dependence of the $\mathrm{HNC}+\mathrm{H} \rightarrow \mathrm{HCN}+\mathrm{H}$ reaction. They found the $\mathrm{HNC} / \mathrm{HCN}$ ratio to be $\sim 1$ at temperatures of $\sim 10 \mathrm{~K}$ and noted that the ratio decreases in OMC-1 as the gas temperature increases from $\sim 10$ to $\sim 150 \mathrm{~K}$. Schilke et al. (1992) then assumed activation energies for both the $\mathrm{HNC}+\mathrm{H}$ and $\mathrm{HNC}+\mathrm{O}$ reactions of $\sim 200 \mathrm{~K}$ in order to reproduce the observed ratios. Using gas-grain and gas-phase chemical modeling, Graninger et al. (2014) furthered the study of $\mathrm{HNC} / \mathrm{HCN}$ in OMC-1, where they identified the critical temperature range of the gas cloud at which the $\mathrm{HNC} / \mathrm{HCN}$ ratio would begin to decrease from unity, i.e. $20-40 \mathrm{~K}$, and placed upper limits on the activation barrier energy of well below $1200 \mathrm{~K}$.

The conclusions from these foregoing observational studies are broadly consistent with those of Jin et al. (2015), who analyzed the HNC/HCN ratio across dark clouds, protostellar objects, and HiI regions. Jin et al. (2015) found the mean ratio decreased gradually across these evolutionary stages. They concluded that the $\mathrm{HNC} / \mathrm{HCN}$ ratio can trace the evolutionary stages of massive star formation from cold molecular clouds $(\sim 20 \mathrm{~K})$ to the warmer molecular gas $(\sim 100 \mathrm{~K})$ associated with HII regions generated by young, massive (OB) stars. Similarly, the ring-like vs. centrally peaked morphologies of $\mathrm{HNC}$ and HCN, respectively, within the disk orbiting TW Hya appear to place constraints on the characteristic temperature necessary to efficiently convert $\mathrm{HNC}$ into $\mathrm{HCN}$ at $\sim 25 \mathrm{~K}$ (Graninger et al. 2015).

The abundances of HNC and HCN in PNe can be expected to change due to thermal and kinetic processes as the envelope of AGB ejecta evolves to post-AGB and, with the CSPN reaching ionization temperatures, into PN stages (Schmidt \& Ziurys 2017a). As it is more stable, HCN is favored in the AGB stage where LTE chemistry dominates the inner envelope of the star (Schmidt \& Ziurys 2017a). Herpin et al. (2002) also observe lower abundances of HNC in post-AGB stages and claim this is due to increased ion-molecule interactions. By the proto-PN phase, abundances of HNC and HCN are expected to restabilize to unity (Schmidt \& Ziurys 2017a; Kimura et al. 2012). In more evolved PNe, models indicate the increased ionization fractions should result in enhanced abundances of $\mathrm{HNC}$ relative to $\mathrm{HCN}$ (Bachiller et al. 1997).

\subsubsection{HNC/HCN ratio with CSPN LUV}

Eight PNe were observed in both the HNC and HCN during our molecular line survey. We find that the observed $\mathrm{HNC} / \mathrm{HCN}$ line intensity ratio ranged from 0.03 to 0.72 within the objects sampled (Table 5).

Notably, this analysis has revealed a previously unknown correlation between the $\mathrm{HNC} / \mathrm{HCN}$ line intensity ratio and the $\mathrm{UV}$ luminosity of the central star. In Fig. 15, we plot the HNC/HCN ratios obtained from our $30 \mathrm{~m}$ data, as well as data from other nearby PNe taken from the literature, versus CSPN UV luminosity. The $\mathrm{HNC} / \mathrm{HCN}$ line ratios for the PNe sample decrease from roughly unity to 0.027 , as the CSPN UV luminosity increases. Linear regression for the data for our survey objects (omitting 
Table 5. HNC/HCN integrated line intensity ratios.

\begin{tabular}{lcr}
\hline \hline Object & Integrated line intensity & Uncertainty \\
\hline NGC 7027 & 0.027 & 0.004 \\
NGC 6445 & 0.247 & 0.035 \\
NGC 6720 & 0.366 & 0.052 \\
NGC 6720 Rim & 0.290 & 0.041 \\
NGC 6853 & 0.320 & 0.045 \\
NGC 6772 & 0.365 & 0.052 \\
NGC 7293 & 0.717 & 0.101 \\
NGC 6781 & 0.452 & 0.064 \\
\hline
\end{tabular}

Notes. Integrated line intensity values of the $\mathrm{HNC} / \mathrm{HCN}$ ratio in observed PNe.

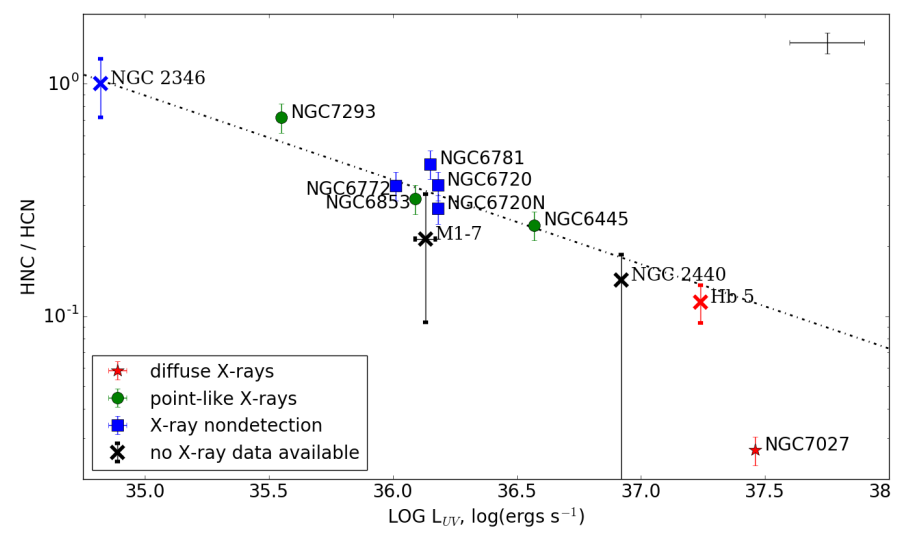

Fig. 15. Flux ratio of $\mathrm{HNC}$ to $\mathrm{HCN}$ plotted against PN central star UV luminosity. Line ratios obtained from observations by Schmidt \& Ziurys (2017a) are denoted by crosses. CSPN UV luminosities from Table 1 and Bachiller et al. (1989b, NGC 2346), Frew (2008, NGC 2440 and $\mathrm{Hb}$ 5), and Sabbadin et al. (1984, M 1-7). The best-fit linear power-law slope (black dashed line, NGC 7027 and literature sources omitted) is $m=-0.363$ with a linear correlation coefficient of $r=-0.885$. Typical uncertainty estimate of $40 \%$ for UV luminosity and $10 \%$ for line ratios is plotted in the top right.

NGC 7027) supports the presence of a power-law relationship between the $\mathrm{HNC} / \mathrm{HCN}$ ratio and CSPN UV luminosity, with a best-fit slope of $m=-0.363$ and a correlation coefficient of $r=-0.885$. There exists no dependence of the $\mathrm{HNC} / \mathrm{HCN}$ ratio on the type or presence of an X-ray source.

For the HNC/HCN line ratio to decrease steadily with UV luminosity, high energy photons must be either heating the gas within the PDR or selectively photodissociating HNC (Aguado et al. 2017). Under the former scenario, as CSPN UV luminosity rises, the resulting CSPN-induced ionization evidently raises the local temperature to exceed the activation energy for conversion of $\mathrm{HNC}$ into $\mathrm{HCN}+\mathrm{H}$. Graninger et al. (2015) suggest that when the local PDR is heated to the range of $25-40 \mathrm{~K}$, the $\mathrm{HNC}+\mathrm{H}$ reaction will lead to a decreased $\mathrm{HNC} / \mathrm{HCN}$ ratio. Under this scenario, PDR heating would therefore be a direct result of photoelectrons generated by UV CSPN emission. Recent observations targeting proto-brown dwarfs revealed a similar decrease in the $\mathrm{HNC} / \mathrm{HCN}$ abundance ratio with bolometric luminosity for the case of protostars (Riaz et al. 2018). Riaz et al. (2018) suggest that this trend is due to the increased efficiency of heating of molecular gas, resulting in declining HNC abundance with increasing protostellar luminosity.

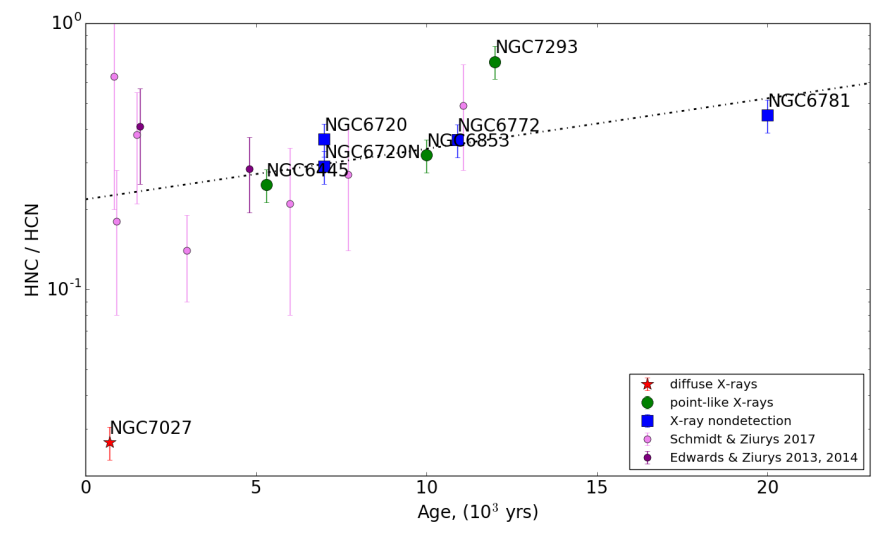

Fig. 16. Flux ratio of $\mathrm{HNC} / \mathrm{HCN}$ plotted against nebular age. Magenta points are PNe observed by Schmidt \& Ziurys (2017a). Their sample encompasses objects with an age range half that of the PN included in our study. Linear regression identified a correlation of $r=0.496$ with a linear slope of $m=0.019$, or an increase in the ratio of $\mathrm{HNC} / \mathrm{HCN}$ of $0.019 \mathrm{kyr}^{-1}$.

Another potential mechanism that might drive the $\mathrm{HNC} / \mathrm{HCN}$ ratio is the difference in photodissociation rates of HNC and HCN. Calculations by Aguado et al. (2017), taking into account the photodissociation cross sections of each molecule as well as variation in the incident UV radiation field, find that selective photodissociation can increase the rate of destruction of $\mathrm{HNC}$ by a factor of $\sim 2-10$ relative to that of $\mathrm{HCN}$. While high effective temperature sources, such as CSPNe, have fewer lowenergy photons and thus a smaller effect on photodissociation rates than cool sources, the interaction between the strength of the evolving UV source and the photochemical reaction rates of $\mathrm{HNC}$ and $\mathrm{HCN}$ could be sufficient to drive the observed trend.

Modeling of HNC and HCN generation in cold, UVirradiated molecular regions is necessary to identify the relevant processes that are most important in determining $\mathrm{HNC} / \mathrm{HCN}$ variation in molecule-rich $\mathrm{PNe}$. In addition, having established the correlation of $\mathrm{HNC} / \mathrm{HCN}$ ratio with UV luminosity for an ensemble of $\mathrm{PNe}$, it is necessary to investigate whether and how the $\mathrm{HNC} / \mathrm{HCN}$ ratio depends on the local UV flux within individual objects.

Note that, since excitation of $\mathrm{HCN}$ and $\mathrm{HNC}$ is similar under similar physical conditions, we expect the trend in their line intensity ratio apparent in Fig. 15 (and the weaker trend in Fig. 16; see Sect. 4.1.2) to closely track a similar trend in their column density ratio.

\subsection{2. $\mathrm{HNC} / \mathrm{HCN}$ ratio with nebular age}

In the past, Herpin et al. (2002) suggested that the emergence of a strong UV source early in a PN's history generates a significant drop in $\mathrm{HCN}$ with respect to $\mathrm{HNC}$ through PDR processing. Recent work on the $\mathrm{HNC} / \mathrm{HCN}$ ratio in young PNe found that there is insignificant change with dynamical age (Schmidt \& Ziurys 2017a). These results were taken to indicate that the ratio does not vary significantly with nebular evolutionary state, and after the proto-PN stage, remains fixed. Because our survey samples PNe spanning a somewhat larger range of dynamical age, we have revisited the question of whether $\mathrm{HNC} / \mathrm{HCN}$ correlates with $\mathrm{PN}$ age (Fig. 16). Linear regression to our $30 \mathrm{~m}$ survey data (omitting NGC 7027) yields a correlation coefficient $r=0.496$, suggesting a marginal correlation. When including data from the literature (pink and purple dots in Fig. 16) (Schmidt \& Ziurys 2017a; Edwards \& Ziurys 2014, 2013), however, we find no 


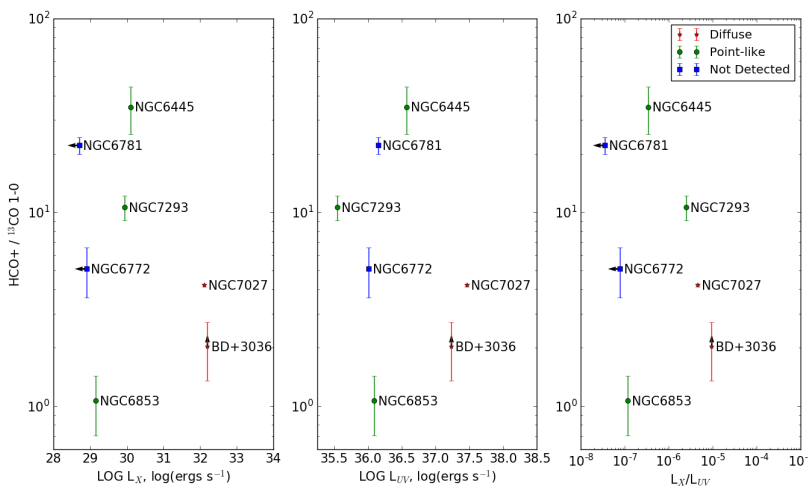

Fig. 17. $\mathrm{HCO}^{+}$to ${ }^{13} \mathrm{CO}(1-0)$ line ratio plotted against the central star's luminosities $L_{\mathrm{X}}, L_{\mathrm{UV}}$, and $L_{\mathrm{X}} / L_{\mathrm{UV}}$. Arrows indicate upper limits.

correlation between $\mathrm{HNC} / \mathrm{HCN}$ ratio and PN age. Nevertheless, the trend obtained from our data appears to match reasonably well with these previous PN survey data. These results support the notion that a continued rise in HNC with respect to $\mathrm{HCN}$ occurs throughout PN evolution. Due to the nature of PN age estimates, however, uncertainties in the ages are difficult to define and thus have not been included in the plot. Additional $\mathrm{HNC}$ and $\mathrm{HCN}$ observations of evolved PNe are necessary to confirm the tentative trend indicated in Fig. 16.

\subsection{Diagnostic of $X$-irradiated gas}

We investigated several potential tracers of X-ray-induced chemistry among the molecular emission lines. Line ratios were compared against X-ray and UV luminosity of each central star, as well as the ratio of the X-ray and UV luminosities. No significant trends emerged from this analysis. We focus here on $\mathrm{HCO}^{+}$, which has long been proposed as a diagnostic of $\mathrm{X}$-irradiation of molecular gas (Deguchi et al. 1990). Specifically, $\mathrm{HCO}^{+}$is generated via $\mathrm{X}$-ray ionization of molecular gas through the reaction $\mathrm{CO}^{+}+\mathrm{H}_{2} \rightarrow \mathrm{HCO}^{+}+\mathrm{H}$ or by the molecular ion $\mathrm{H}_{3}^{+}$through the reaction $\mathrm{H}_{3}^{+}+\mathrm{CO} \rightarrow \mathrm{HCO}^{+}+\mathrm{H}_{2}$ (Zhang et al. 2008). An enhanced abundance of $\mathrm{HCO}^{+}$may then act as a tracer for X-ray emission in the PNe, and we would expect line ratios correlated with CSPN or nebular X-ray luminosity.

Figure 17 plots the ratio of $\mathrm{HCO}^{+}$to ${ }^{13} \mathrm{CO}$ against CSPN UV and X-ray emission. ${ }^{13} \mathrm{CO}$ was chosen as it acts as a proxy of the molecular mass and is not coupled to X-irradiation. The absence of a clear trend in the left-hand panel of Fig. 17 may suggest that X-rays are not directly driving $\mathrm{HCO}^{+}$production in $\mathrm{PNe}$. On the other hand, Fig. 17 (center panel) also does not reveal a clear trend of enhanced $\mathrm{HCO}^{+}$in the presence of strong UV from CSPNe. Hence, it remains to establish the irradiation source that is primarily responsible for the production of this molecular ion in $\mathrm{PNe}$.

\section{3. $\mathrm{CO}^{+}$and $\mathrm{N}_{2} \mathrm{H}^{+}$}

In PNe with hot central stars or high energy emission, X-rays have the potential to drive the chemical pathways available to the molecular gas. $\mathrm{CO}^{+}$is produced in higher abundances in the presence of $\mathrm{X}$-ray dominated regions through the reaction $\mathrm{C}^{+}+\mathrm{OH} \rightarrow \mathrm{CO}^{+}+\mathrm{H}$ (Spaans \& Meijerink 2007). An enhanced abundance of $\mathrm{CO}^{+}$then might suggest formation through this $\mathrm{X}$-ray driven reaction. The main chemical pathway for the production of $\mathrm{N}_{2} \mathrm{H}^{+}$, i.e., $\mathrm{H}_{3}^{+}+\mathrm{N}_{2} \rightarrow \mathrm{N}_{2} \mathrm{H}^{+}+\mathrm{H}_{2}$, also requires X-ray or cosmic ray irradiation as a means to generate the requisite $\mathrm{H}_{3}^{+}$ion. (Zhang et al. 2008; Bell et al. 2007).
Of the nine observed PNe, transitions of $\mathrm{CO}^{+}$and $\mathrm{N}_{2} \mathrm{H}^{+}$were detected only in the spectra of NGC 7027. None of the other objects surveyed here have had confirmed detections of $\mathrm{CO}^{+}$, with the possible exception of NGC 6781, despite the potential importance of $\mathrm{CO}^{+}$in the generation of $\mathrm{HCO}^{+}$(Bell et al. 2007). Considering the $\mathrm{CO}^{+}$line was quite weak in NGC 7027 compared to ${ }^{12} \mathrm{CO}$, the molecule may be present in other $\mathrm{X}$-ray emitting $\mathrm{PNe}$, but at a level below our survey sensitivity. Given that NGC 7027 harbors an unusually luminous diffuse $\mathrm{X}$-ray source and an unusually large mass of molecular gas, these detections hint at the viability of $\mathrm{CO}^{+}$and $\mathrm{N}_{2} \mathrm{H}^{+}$diagnostics of $\mathrm{X}$-irradiation of molecular gas. Shocks may also play a role in $\mathrm{N}_{2} \mathrm{H}^{+}$formation, however, and future observations are required to distinguish these two mechanisms.

\section{Summary}

This study presents observations of key molecular transitions across the $88-236 \mathrm{GHz}$ range toward nine planetary nebulae with the IRAM $30 \mathrm{~m}$ radio telescope. We report new detections for four of the PNe in the survey across thirteen molecular lines included in our spectral coverage. Our survey yielded new detections of molecular transitions of $\mathrm{CO}, \mathrm{CN}, \mathrm{HCN}, \mathrm{HNC}$, and/or $\mathrm{HCO}^{+}$in NGC 6445, NGC 6853, and NGC 6772. The molecular ion $\mathrm{HCO}^{+}$was also detected in $\mathrm{BD}+30^{\circ} 3639$ for the first time. In addition, confirmation of previously detected molecules were made in NGC 7027, NGC 6720, NGC 7293, and NGC 6781.

Our analysis of line ratios that are potential diagnostics of high-energy irradiation has revealed a strong but previously unrecognized anticorrelation between the $\mathrm{HNC} / \mathrm{HCN}$ line intensity ratio and CSPN UV luminosity. This anticorrelation, which persists over nearly 3 orders of magnitude in central star UV luminosity, provides strong evidence that $\mathrm{HNC} / \mathrm{HCN}$ acts as a tracer for heating of the nebular molecular gas by UV photons. Our survey additionally found a marginal correlation between the $\mathrm{HNC} / \mathrm{HCN}$ line ratio and $\mathrm{PN}$ age.

Other irradiation tracers explored in our PN molecular line survey yield more ambiguous results. Though predicted to increase in abundance with $\mathrm{X}$-irradiation, we find the intensity of $\mathrm{HCO}^{+}$line emission in $\mathrm{PNe}$ shows no clear correlation with PN X-ray luminosity. Detections of $\mathrm{CO}^{+}$and $\mathrm{N}_{2} \mathrm{H}^{+}$in our survey were limited to the particularly molecule-rich and luminous NGC 7027, which had been previously detected in lines of both species. This suggests that $\mathrm{CO}^{+}$and $\mathrm{N}_{2} \mathrm{H}^{+}$trace especially intense high-energy irradiation of molecular gas.

Acknowledgements. This work was supported in part by a subcontract issued to RIT under NASA ADAP grant \#80NSSC17K0057 to STScI (PI: B. Sargent), and by the Spanish MINECO within the program AYA2016-78994-P. It is also based on observations carried out with the IRAM telescopes. IRAM is supported by INSU/CNRS (France), MPG (Germany) and IGN (Spain). J.B. wishes to acknowledge useful discussions with Pierre Hily-Blant and Thierry Forveille during his Fall 2018 residency at IPAG under the support of the Chateaubriand Fellowship of the Office for Science \& Technology of the Embassy of France in the United States.

\section{References}

Aguado, A., Roncero, O., Zanchet, A., Agúndez, M., \& Cernicharo, J. 2017, ApJ, 838, 33

Akras, S., \& Steffen, W. 2011, MNRAS, 423, 925

Aleman, I., Ueta, T., Ladjal, D., et al. 2014, A\&A, 566, A79

Ali, A., Sabin, L., Snaid, S., \& Basurah, H. M. 2012, A\&A, 541, A98

Bachiller, R., Bujarrabal, V., Martin-Pintado, J., \& Gómez-González, J. 1989a, A\&A, 218, 252

Bachiller, R., Planesas, P., Martin-Pintado, J., Bujarrabal, V., \& Tafalla, M. 1989b, A\&A, 210, 366 
Bachiller, R., Huggins, P., Cox, P., \& Forveille, T. 1991, A\&A, 247, 525

Bachiller, R., Forveille, T., Huggins, P., \& Cox, P. 1997, A\&A, 324, 1123

Balick, B., \& Frank, A. 2002, ARA\&A, 40, 439

Bell, T. A., Whyatt, W., Viti, S., \& Redman, M. P. 2007, MNRAS, 382, 1139

Blöcker, T. 2001, Astrophys. Space Sci., 275, 1

Bujarrabal, V., Castro-Carrizo, A., Alcolea, J., \& Contreras, C. S. 2001, A\&A, 377,868

Cox, P., Huggins, P., Maillard, J.-P., et al. 2002, A\&A, 384, 603

Deguchi, S., Izumiura, H., Kaifu, N., et al. 1990, ApJ, 351, 522

De Marco, O. D., \& Izzard, R. G. 2017, PASA, 34, 35

Edwards, J. L., \& Ziurys, L. M. 2013, ApJ, 770, L5

Edwards, J. L., \& Ziurys, L. M. 2014, ApJ, 794, L27

Edwards, J. L., Cox, E., \& Ziurys, L. 2014, AJ, 791, 79

Freeman, M. J., \& Kastner, J. H. 2016, ApJS, 226, 15

Freeman, M., Montez, R., Jr., Kastner, J. H., et al. 2014, ApJ, 794, 99

Frew, D. J. 2008, Ph.D. Thesis, Macquarie University, NSW, Australia

Frew, D. J., Parker, Q. A., \& Bojičić, I. S. 2016, MNRAS, 455, 1459

Gaia Collaboration (Brown, A. G. A., et al.) 2018, A\&A, 616, A1

Górny, S. K., Stasińnska, G., \& Tylenda, R. 1997, A\&A, 318, 256

Graham, J. R., Serabyn, E., Herbst, T. M., et al. 1993, AJ, 105, 250

Graninger, D. M., Herbst, E., Öberg, K. I., \& Vasyunin, A. I. 2014, ApJ, 787, 74

Graninger, D., Öberg, K. I., Qi, C., \& Kastner, J. 2015, ApJ, 807, L15

Guerrero, M. A., Chu, Y.-H., Gruendl, R. A., Williams, R. M., \& Kaler, J. B. 2001, ApJ, 553, L55

Gussie, G. T., \& Taylor, A. 1995, MNRAS, 273, 801

Hasegawa, T. I., \& Kwok, S. 2001, ApJ, 562, 824

Herpin, F., Goicoechea, J. R., Pardo, J. R., \& Cernicharo, J. 2002, ApJ, 577, 961

Huarte-Espinosa, M., Frank, A., Balick, B., et al. 2012, MNRAS, 424, 2055

Huggins, P., \& Healy, A. P. 1989, ApJ, 346, 201

Huggins, P., Bachiller, R., Cox, P., \& Forveille, T. 1992, ApJ, 401, L43

Huggins, P., Bachiller, R., Cox, P., \& Forveille, T. 1996, A\&A, 315, 284

Jin, M., Lee, J.-E., \& Kim, K.-T. 2015, ApJ, 219, 1

Kahane, C., Cernicharo, J., Gómez-González, J., \& Guélin, M. 1992, A\&A, 256, 235

Kastner, J. H., Weintraub, D. A., Gatley, I., Merrill, K. M., \& Probst, R. G. 1996, ApJ, 462, 777
Kastner, J. H., Vrtilek, S. D., \& Soker, N. 2001, ApJ, 550, L189 Kastner, J. H., Montez Jr. R., Balick, B., et al. 2012, AJ, 144, 58 Kimura, R. K., Gruenwald, R., \& Aleman, I. 2012, A\&A, 541, A112 Latter, W. B., Dayal, A., Bieging, J. H., et al. 2000, ApJ, 539, 783

Li, J., Harrington, J. P., \& Borkowski, K. J. 2002, AJ, 123, 2676

Montez, Jr. R., Kastner, J. H., Balick, B., et al. 2015, ApJ, 800, 8 Müller, H. S. P., Thorwirth, S., Roth, D. A., \& Winnewisser, G. 2001, A\&A, 370 L49

Müller, H. S. P., Schlöder, F., Stutzki, J., \& Winnewisser, G. 2005, J. Mol. Struct., 742,215

O’Dell, C. R., Balick, B., Hajian, A. R., Henney, W. J., \& Burkert, A. 2002, AJ, 123,3329

O’Dell, C. R., Sabbadin, F., \& Henney, W. J. 2007, AJ, 134, 1679

Phillips, J. P. 1984, A\&A, 137, 92

Riaz, B., Thi, W.-F., \& Caselli, P. 2018, MNRAS, 481, 4662

Sabbadin, F., Falomo, R., \& Ortolani, S. 1984, A\&A, 137, 177

Sahai, R., Morris, M. R., \& Villar, G. G. 2011, AJ, 141, 134

Salama, A., Kessler, M. F., Leech, K., \& Schulz, B., 2000, An Infrared/ Millimeter Study of the Dumbbell Nebula, ESA-SP, 456

Santander-García, M., Bujarrabal, V., \& Alcolea, J. 2012, A\&A, 545, A114

Schilke, P., Walmsley, C. M., des Forets, G. P., et al. 1992, A\&A, 256, 595

Schmidt, D. R., \& Ziurys, L. M. 2016, ApJ, 817, 175

Schmidt, D. R., \& Ziurys, L. M. 2017a, ApJ, 835, 79

Schmidt, D. R., \& Ziurys, L. M. 2017b, ApJ, 850, 123

Schmidt, D. R., Zack, L. N., \& Ziurys, L. M. 2018, ApJ, 864, 31

Spaans, M., \& Meijerink, R. 2007, ApJ, 664, L23

Stanghellini, L., \& Shaw, R. A. 2008, ApJ, 689, 194

Sun, J., \& Sun, Y. 2000, Sci. China, 43, 217

Tielens, A. G. G. M., \& Hollenbach, D. J. 1985, ApJ, 291, 722

Ueta, T., Ladjal, D., Exter, K. M., et al. 2014, A\&A, 565, A36

Young, K., Cox, P., Huggins, P., Forveille, T., \& Bachiller, R. 1999, ApJ, 522, 387

Zack, L. N., \& Ziurys, L. M. 2013, ApJ, 765, 112

Zeigler, N. R., Zack, L. N., Woolf, N. J., \& Ziurys, L. M. 2013, ApJ, 778, 16

Zhang, Y., Kwok, S., \& Dinh-V-Trung. 2008, ApJ, 678, 328

Zuckerman, B., \& Gatley, I. 1988, ApJ, 324, 501

Zuckerman, B., Kastner, J. H., \& Gatley, I. 1990, ApJ, 356, L59 


\section{Appendix A: Additional tables}

Table A.1. PNe previously surveyed in molecular lines.

\begin{tabular}{|c|c|c|c|c|c|c|}
\hline $\mathrm{PNe}$ & Species & $\begin{array}{c}T_{\mathrm{mb}}\left(\sigma_{\mathrm{mb}}\right) \\
(\mathrm{K})\end{array}$ & $\begin{array}{c}\int T_{\mathrm{mb}} \mathrm{d} V\left(\sigma_{\text {area }}\right) \\
\left(\mathrm{K} \mathrm{km} \mathrm{s}^{-1}\right)\end{array}$ & $\begin{array}{c}\text { Prev. work } \\
T_{\mathrm{mb}}\end{array}$ & $\begin{array}{l}\text { Prev. work } \\
\int T_{\mathrm{mb}} \mathrm{d} V\end{array}$ & References \\
\hline \multirow[t]{13}{*}{ NGC 7027} & $\mathrm{CO}(1-0)$ & $9.6(0.03)$ & $267.0(0.14)$ & $11.9(-)$ & $332(-)$ & $\mathrm{a}$ \\
\hline & $\mathrm{CO}(2-1)$ & $26.2(0.05)$ & $520.0(0.15)$ & $30.9(-)$ & $667(-)$ & $\mathrm{a}$ \\
\hline & ${ }^{13} \mathrm{CO}(1-0)$ & $0.28(0.01)$ & $6.68(0.06)$ & $0.3(-)$ & $6.4(0.3)$ & $a, b$ \\
\hline & ${ }^{13} \mathrm{CO}(2-1)$ & $0.89(0.01)$ & $22.4(0.09)$ & $1.3(-)$ & $17.3(0.8)$ & $a, b$ \\
\hline & $\mathrm{C}^{17} \mathrm{O}(1-0)$ & $0.045(0.031)$ & $1.51(0.18)$ & $-(-)$ & $0.92(0.12)$ & $\mathrm{c}$ \\
\hline & $\mathrm{C}^{17} \mathrm{O}(2-1)$ & $0.268(0.081)$ & $3.33(0.354)$ & $0.032(-)$ & $1.87(0.08)$ & $\mathrm{d}, \mathrm{c}$ \\
\hline & $\mathrm{CO}^{+}(2-1)$ & $0.05(0.01)$ & $2.18(0.11)$ & $0.020(-)$ & $0.87(0.13)$ & $\mathrm{e}$ \\
\hline & $\mathrm{N}_{2} \mathrm{H}^{+}(1-0)$ & $0.08(0.01)$ & $0.640(0.020)$ & $0.017(-)$ & $0.35(-)$ & $\mathrm{d}$ \\
\hline & $\mathrm{HCO}^{+}(1-0)$ & $1.36(0.009)$ & $28.1(0.05)$ & $0.247(-)$ & $26.5(0.2)$ & $d, b$ \\
\hline & CN (2-1) & 1.47 (0.016) & $86.5(0.12)$ & $0.028(-)$ & $41.9(0.6)$ & $d, b$ \\
\hline & $\mathrm{CN}(1-0)$ & $0.543(0.038)$ & $6.95(0.048)$ & $-(-)$ & $25.1(0.4)$ & $\mathrm{b}$ \\
\hline & $\mathrm{HCN}(1-0)$ & $0.35(0.005)$ & $11.6(0.03)$ & $0.059(-)$ & $14.3(0.4)$ & $d, b$ \\
\hline & HNC (1-0) & $0.034(0.006)$ & $0.309(0.022)$ & $-(-)$ & $1.0(1.2)$ & $\mathrm{b}$ \\
\hline \multirow[t]{11}{*}{ NGC 6720} & $\mathrm{CO}(1-0)$ & $-(-)$ & $-(-)$ & $0.047(0.005)$ & $-(-)$ & $\mathrm{f}$ \\
\hline & $\mathrm{CO}(2-1)$ & $-(-)$ & $-(-)$ & $0.023(0.004)$ & $20.0(0.4)$ & $f, b$ \\
\hline & ${ }^{13} \mathrm{CO}(1-0)$ & $-(-)$ & $-(-)$ & $-(-)$ & $0.27(0.11)$ & $\mathrm{b}$ \\
\hline & ${ }^{13} \mathrm{CO}(2-1)$ & $0.156(0.008)$ & $1.30(0.03)$ & $-(-)$ & $0.9(0.3)$ & $\mathrm{b}$ \\
\hline & $\mathrm{CO}^{+}(2-1)$ & $<0.021(0.007)$ & $<0.11(0.04)$ & $-(-)$ & $-(-)$ & \\
\hline & $\mathrm{N}_{2} \mathrm{H}^{+}(1-0)$ & $<0.014(0.006)$ & $<0.012(0.004)$ & $-(-)$ & $-(-)$ & \\
\hline & $\mathrm{HCO}^{+}(1-0)$ & $0.103(0.004)$ & $1.760(0.019)$ & $0.019(0.002)$ & $0.86(0.11)$ & $f, b$ \\
\hline & CN (2-1) & $0.31(0.01)$ & $10.10(0.06)$ & $-(-)$ & $4.5(0.7)$ & $\mathrm{b}$ \\
\hline & $\mathrm{CN}(1-0)$ & $-(-)$ & $-(-)$ & $-(-)$ & $3.9(0.3)$ & $\mathrm{b}$ \\
\hline & $\mathrm{HCN}(1-0)$ & $0.07(0.004)$ & $1.83(0.02)$ & $-(-)$ & $3.2(0.2)$ & b \\
\hline & HNC (1-0) & $0.04(0.004)$ & $0.67(0.02)$ & $-(-)$ & $0.74(0.10)$ & b \\
\hline \multirow[t]{11}{*}{ NGC 6720 Rim } & $\mathrm{CO}(1-0)$ & $-(-)$ & $-(-)$ & $0.047(0.005)$ & $-(-)$ & $\mathrm{f}$ \\
\hline & $\mathrm{CO}(2-1)$ & $-(-)$ & $-(-)$ & $0.023(0.004)$ & $20.0(0.4)$ & $f, b$ \\
\hline & ${ }^{13} \mathrm{CO}(1-0)$ & $-(-)$ & $-(-)$ & $-(-)$ & $0.27(0.11)$ & $\mathrm{b}$ \\
\hline & ${ }^{13} \mathrm{CO}(2-1)$ & $0.05(0.01)$ & $0.57(0.06)$ & $-(-)$ & $0.9(0.3)$ & $\mathrm{b}$ \\
\hline & $\mathrm{CO}^{+}(2-1)$ & $<0.027(0.009)$ & $<0.18(0.06)$ & $-(-)$ & $-(-)$ & \\
\hline & $\mathrm{N}_{2} \mathrm{H}^{+}(1-0)$ & $<0.014(0.006)$ & $<0.017(0.04)$ & $-(-)$ & $-(-)$ & \\
\hline & $\mathrm{HCO}^{+}(1-0)$ & $0.063(0.005)$ & $1.28(0.03)$ & $0.019(0.002)$ & $0.86(0.11)$ & $f, b$ \\
\hline & CN (2-1) & $0.29(0.01)$ & $11.1(0.08)$ & $-(-)$ & $4.5(0.7)$ & $\mathrm{b}$ \\
\hline & CN (1-0) & $-(-)$ & - (一) & $-(-)$ & $3.9(0.3)$ & b \\
\hline & $\mathrm{HCN}(1-0)$ & $0.074(0.005)$ & $2.08(0.03)$ & $-(-)$ & $3.2(0.2)$ & $\mathrm{b}$ \\
\hline & HNC (1-0) & $0.027(0.005)$ & $0.603(0.027)$ & $-(-)$ & $0.74(0.10)$ & $\mathrm{b}$ \\
\hline \multirow[t]{11}{*}{ NGC 7293} & $\mathrm{CO}(1-0)$ & $0.66(0.02)$ & $6.0(0.06)$ & $0.17(0.030)$ & $-(-)$ & $\mathrm{g}$ \\
\hline & $\mathrm{CO}(2-1)$ & $2.05(0.06)$ & $12.3(0.1)$ & $0.40(0.050)$ & $17.5(0.7)$ & $\mathrm{g}, \mathrm{b}$ \\
\hline & ${ }^{13} \mathrm{CO}(1-0)$ & $0.044(0.009)$ & $0.096^{(\star)}(0.014)$ & $-(-)$ & $0.67(0.09)$ & $\mathrm{b}$ \\
\hline & ${ }^{13} \mathrm{CO}(2-1)$ & $0.047(0.006)$ & $0.60(0.03)$ & $-(-)$ & $1.8(0.2)$ & b \\
\hline & $\mathrm{CO}^{+}(2-1)$ & $<0.021(0.007)$ & $<0.07(0.02)$ & $-(-)$ & $-(-)$ & \\
\hline & $\mathrm{N}_{2} \mathrm{H}^{+}(1-0)$ & $<0.001(0.004)$ & $-(-)$ & $-(-)$ & $-(-)$ & \\
\hline & $\mathrm{HCO}^{+}(1-0)$ & $0.088(0.044)$ & $1.020(0.017)$ & $0.04(0.008)$ & $0.89(0.10)$ & $\mathrm{g}, \mathrm{b}$ \\
\hline & $\mathrm{CN}(2-1)$ & $0.029(0.009)$ & $0.18(0.02)$ & $-(-)$ & $1.0(0.2)$ & $\mathrm{b}$ \\
\hline & $\mathrm{CN}(1-0)$ & $0.054(0.008)$ & $0.629(0.04)$ & $-(-)$ & $2.7(0.2)$ & $\mathrm{b}$ \\
\hline & $\mathrm{HCN}(1-0)$ & $0.041(0.003)$ & $0.649(0.014)$ & $-(-)$ & $1.6(0.2)$ & $\mathrm{b}$ \\
\hline & HNC (1-0) & $0.038(0.003)$ & $0.465(0.013)$ & $-(-)$ & $0.80(0.11)$ & b \\
\hline \multirow[t]{11}{*}{ NGC 6781} & $\mathrm{CO}(1-0)$ & $1.14(0.02)$ & $15.3(0.07)$ & $0.35(-)$ & - (一) & $\mathrm{h}$ \\
\hline & $\mathrm{CO}(2-1)$ & $5.26(0.05)$ & $49.6(0.11)$ & $-(-)$ & $28.4(0.6)$ & b \\
\hline & ${ }^{13} \mathrm{CO}(1-0)$ & $0.049(0.009)$ & $0.159(0.016)$ & $-(-)$ & $0.32(0.14)$ & $\mathrm{b}$ \\
\hline & ${ }^{13} \mathrm{CO}(2-1)$ & $0.203(0.007)$ & $2.21(0.03)$ & $-(-)$ & $1.7(0.2)$ & $\mathrm{b}$ \\
\hline & $\mathrm{CO}^{+}(2-1)$ & $<0.021(0.007)$ & $<0.09(0.03)$ & $0.031(0.008)$ & $0.249(-)$ & $\mathrm{i}$ \\
\hline & $\mathrm{N}_{2} \mathrm{H}^{+}(1-0)$ & $<0.011(0.003)$ & - (一) & $-(-)$ & $-(-)$ & \\
\hline & $\mathrm{HCO}^{+}(1-0)$ & $0.167(0.003)$ & $3.520(0.018)$ & $-(-)$ & $2.2(0.2)$ & b \\
\hline & $\mathrm{CN}(2-1)$ & $0.40(0.01)$ & $13.2(0.06)$ & $-(-)$ & $4.6(0.5)$ & b \\
\hline & CN (1-0) & $0.317(0.042)$ & $5.51(0.33)$ & $-(-)$ & $7.0(0.4)$ & $\mathrm{b}$ \\
\hline & $\mathrm{HCN}(1-0)$ & $0.16(0.003)$ & $3.83(0.02)$ & $-(-)$ & $2.4(0.2)$ & $\mathrm{b}$ \\
\hline & HNC (1-0) & $0.08(0.003)$ & $1.73(0.02)$ & $-(-)$ & $1.65(0.15)$ & $\mathrm{b}$ \\
\hline
\end{tabular}

Notes. ${ }^{(\star)}$ Uncertain.

References. a: Santander-García et al. (2012); b: Bachiller et al. (1997); c: Kahane et al. (1992); d: Zhang et al. (2008); e: Hasegawa \& Kwok (2001); f: Edwards et al. (2014); g: Zack \& Ziurys (2013); h: Zuckerman et al. (1990); i: Bell et al. (2007). 
Table A.2. PNe not previously surveyed in molecular lines.

\begin{tabular}{|c|c|c|c|c|c|c|}
\hline $\mathrm{PNe}$ & Species & $\begin{array}{c}T_{\mathrm{mb}}\left(\sigma_{\mathrm{mb}}\right) \\
(\mathrm{K})\end{array}$ & $\begin{array}{c}\int T_{\mathrm{mb}} \mathrm{d} V\left(\sigma_{\text {area }}\right) \\
\left(\mathrm{K} \mathrm{km} \mathrm{s}^{-1}\right)\end{array}$ & $\begin{array}{c}\text { Prev. work } \\
T_{\mathrm{mb}} \\
\end{array}$ & $\begin{array}{l}\text { Prev. work } \\
\int T_{\mathrm{mb}} \mathrm{d} V\end{array}$ & References \\
\hline $\mathrm{BD}+30^{\circ} 3639$ & $\begin{array}{l}\mathrm{CO}(1-0) \\
\mathrm{CO}(2-1) \\
{ }^{13} \mathrm{CO}(1-0) \\
{ }^{13} \mathrm{CO}(2-1) \\
\mathrm{CO}^{+}(2-1) \\
\mathrm{HCO}^{+}(1-0) \\
\mathrm{CN}(2-1) \\
\mathrm{CN}(1-0) \\
\mathrm{HCN}(1-0) \\
\mathrm{HNC}(1-0)\end{array}$ & $\begin{array}{c}<0.39(0.13) \\
<1.2(0.4) \\
<0.09(0.03) \\
<0.018(0.006) \\
<0.018(0.006) \\
0.029(0.003) \\
<0.027(0.009) \\
<0.1(0.05) \\
<0.009(0.003) \\
<0.009(0.003)\end{array}$ & $\begin{array}{c}<2.5(0.8) \\
<5.6(1.8) \\
<0.57(0.19) \\
<0.17(0.06) \\
<0.16(0.05) \\
1.16(0.02) \\
<0.17(0.06) \\
-(-) \\
<0.07(0.02) \\
<0.06(0.02)\end{array}$ & $\begin{array}{l}-(-) \\
\leq 0.20(-) \\
-(-) \\
-(-) \\
-(-) \\
-(-) \\
-(-) \\
-(-) \\
-(-) \\
-(-)\end{array}$ & $\begin{array}{l}5.2(-) \\
4.7(-) \\
-(-) \\
-(-) \\
-(-) \\
-(-) \\
-(-) \\
-(-) \\
-(-) \\
-(-)\end{array}$ & $\stackrel{\mathrm{a}}{\mathrm{b}, \mathrm{a}}$ \\
\hline NGC 6445 & $\begin{array}{l}\mathrm{CO}(1-0) \\
\mathrm{CO}(2-1) \\
{ }^{13} \mathrm{CO}(1-0) \\
{ }^{13} \mathrm{CO}(2-1) \\
\mathrm{CO}^{+}(2-1) \\
\mathrm{N}_{2} \mathrm{H}^{+}(1-0) \\
\mathrm{HCO}^{+}(1-0) \\
\mathrm{CN}(2-1) \\
\mathrm{CN}(1-0) \\
\mathrm{HCN}(1-0) \\
\mathrm{HNC}(1-0)\end{array}$ & $\begin{array}{c}0.23(0.03) \\
1.24(0.07) \\
0.03(0.01) \\
0.32(0.006) \\
<0.021(0.007) \\
0.006(0.002) \\
0.052(0.003) \\
0.257(0.009) \\
0.362(0.044) \\
0.115(0.003) \\
0.034(0.003)\end{array}$ & $\begin{array}{c}6.36(0.14) \\
14.8(0.17) \\
0.051^{(\star)}(0.014) \\
0.93(0.05) \\
<0.1(0.03) \\
0.025(0.007) \\
1.78(0.02) \\
11.2(0.06) \\
2.74(0.21) \\
4.54(0.02) \\
1.12(0.02)\end{array}$ & $\begin{array}{l}0.25(-) \\
0.25(-) \\
-(-) \\
-(-) \\
-(-) \\
-(-) \\
-(-) \\
-(-) \\
-(-) \\
-(-) \\
-(-)\end{array}$ & $\begin{array}{l}9.29(-) \\
11.1(-) \\
-(-) \\
-(-) \\
-(-) \\
-(-) \\
-(-) \\
-(-) \\
-(-) \\
-(-) \\
-(-)\end{array}$ & $\begin{array}{c}\mathrm{c} \\
\mathrm{d}, \mathrm{c}\end{array}$ \\
\hline NGC 7008 & $\begin{array}{l}\mathrm{CO}(1-0) \\
\mathrm{CO}(2-1) \\
{ }^{13} \mathrm{CO}(1-0) \\
{ }^{13} \mathrm{CO}(2-1) \\
\mathrm{CO}^{+}(2-1) \\
\mathrm{N}_{2} \mathrm{H}^{+}(1-0) \\
\mathrm{HCO}^{+}(1-0) \\
\mathrm{CN}(2-1) \\
\mathrm{CN}(1-0) \\
\mathrm{HCN}(1-0) \\
\mathrm{HNC}(1-0)\end{array}$ & $\begin{array}{c}1.15(0.03) \\
1.64(0.06) \\
0.057(0.009) \\
0.04(\star \star)(0.02) \\
<0.06(0.02) \\
<0.01(0.006) \\
0.013(0.005) \\
<0.06(0.02) \\
<0.016(0.008) \\
<0.015(0.005) \\
<0.015(0.005)\end{array}$ & $\begin{array}{c}2.56(0.04) \\
2.09^{(\star)}(0.05) \\
0.115^{(\star)}(0.013) \\
0.55^{(\star)}(0.09) \\
<0.08(0.03) \\
-(-) \\
0.113(0.015) \\
<0.07(0.02) \\
<0.082(0.025) \\
<0.019(0.006) \\
<0.019(0.006)\end{array}$ & $\begin{array}{c}-(-) \\
-(-) \\
-(-) \\
-(-) \\
-(-) \\
-(-) \\
<0.004(-) \\
-(-) \\
-(-) \\
<0.006(-) \\
-(-)\end{array}$ & $\begin{array}{l}-(-) \\
-(-) \\
-(-) \\
-(-) \\
-(-) \\
-(-) \\
-(-) \\
-(-) \\
-(-) \\
-(-) \\
-(-)\end{array}$ & $\mathrm{e}$ \\
\hline NGC 6853 & $\begin{array}{l}\mathrm{CO}(1-0) \\
\mathrm{CO}(2-1) \\
{ }^{13} \mathrm{CO}(1-0) \\
{ }^{13} \mathrm{CO}(2-1) \\
\mathrm{CO}^{+}(2-1) \\
\mathrm{N}_{2} \mathrm{H}^{+}(1-0) \\
\mathrm{HCO}^{+}(1-0) \\
\mathrm{CN}(2-1) \\
\mathrm{CN}(1-0) \\
\mathrm{HCN}(1-0) \\
\mathrm{HNC}(1-0)\end{array}$ & $\begin{array}{c}0.13(0.02) \\
0.36(0.06) \\
<0.03(0.01) \\
0.013(0.004) \\
<0.012(0.004) \\
<0.005(0.002) \\
0.020(0.002) \\
0.072(0.007) \\
0.108(0.027) \\
0.069(0.002) \\
0.030(0.002)\end{array}$ & $\begin{array}{c}1.41(0.07) \\
3.48^{(\star)}(0.14) \\
<0.09(0.03) \\
0.09(0.02) \\
<0.052(0.018) \\
<0.006(0.002) \\
0.096(0.006) \\
1.38^{(\star)}(0.1) \\
0.524(0.053) \\
0.637(0.008) \\
0.204(0.007)\end{array}$ & $\begin{array}{c}0.097(0.012) \\
0.251(0.008) \\
-(-) \\
-(-) \\
-(-) \\
-(-) \\
0.026(0.002) \\
-(-) \\
-(-) \\
-(-) \\
-(-)\end{array}$ & $\begin{array}{l}1.21(0.29) \\
2.49(0.06) \\
-(-) \\
-(-) \\
-(-) \\
-(-) \\
0.33(0.03) \\
-(-) \\
-(-) \\
-(-) \\
-(-)\end{array}$ & $\begin{array}{l}\mathrm{f} \\
\mathrm{f}\end{array}$ \\
\hline NGC 6772 & $\begin{array}{l}\mathrm{CO}(1-0) \\
\mathrm{CO}(2-1) \\
{ }^{13} \mathrm{CO}(1-0) \\
{ }^{13} \mathrm{CO}(2-1) \\
\mathrm{CO}^{+}(2-1) \\
\mathrm{N}_{2} \mathrm{H}^{+}(1-0) \\
\mathrm{HCO}^{+}(1-0) \\
\mathrm{CN}(2-1) \\
\mathrm{CN}(1-0) \\
\mathrm{HCN}(1-0) \\
\mathrm{HNC}(1-0) \\
\end{array}$ & $\begin{array}{c}0.24(0.02) \\
0.55(0.04) \\
<0.021(0.007) \\
0.040(0.007) \\
<0.021(0.007) \\
<0.01(0.004) \\
0.030(0.003) \\
5.89(0.01) \\
0.040(0.010) \\
0.021(0.003) \\
0.012(0.003)\end{array}$ & $\begin{array}{c}2.5(0.05) \\
6.36(0.11) \\
<0.07(0.02) \\
0.32(0.03) \\
<0.1(0.03) \\
<0.005(0.002) \\
0.358(0.012) \\
1.26(0.005) \\
0.329(0.037) \\
0.419(0.016) \\
0.153(0.013)\end{array}$ & $\begin{array}{c}0.03(-) \\
0.05(0.01) \\
-(-) \\
-(-) \\
-(-) \\
<(-) \\
<0.003(-) \\
-(-) \\
-(-) \\
0.010(0.006) \\
0.0027(0.0012)\end{array}$ & $\begin{array}{c}-(-) \\
1.39\left(0.41^{(\star)}\right) \\
-(-) \\
-(-) \\
-(-) \\
-(-) \\
-(-) \\
-(-) \\
-(-) \\
0.299\left(0.247^{(\star)}\right) \\
-(-)\end{array}$ & $\begin{array}{l}\mathrm{e} \\
\mathrm{h}\end{array}$ \\
\hline
\end{tabular}

Notes. ${ }^{(\star)}$ Uncertain.

References. a: Bachiller et al. (1991); b: Gussie \& Taylor (1995); c: Sun \& Sun (2000); d: Huggins \& Healy (1989); e: Schmidt \& Ziurys (2016); f: Edwards et al. (2014); g: Zuckerman et al. (1990); h: Schmidt \& Ziurys (2017a). 